\title{
WEIGHTED KERNEL ESTIMATORS IN NONPARAMETRIC BINOMIAL REGRESSION
}

\author{
Hidenori OKUMURA ${ }^{\dagger}$ and Kanta NAITO $\ddagger$ \\ ${ }^{\dagger}$ Department of Business management and information science, Chugoku Ju- \\ nior College, Okayama, 701-0197 Japan \\ ‡Department of Mathematics, Shimane University, Matsue, 690-8504 Japan
}

\begin{abstract}
This paper is concerned with nonparametric binomial regression. Two kernel-based binomial regression estimators and their bias-adjusted versions are proposed, whose kernels are weighted by the inverses of variance estimators of the observed proportion at each covariate. Asymptotic theories for deriving asymptotic mean squared errors (AMSEs) of proposed estimators are developed. Comparisons with other estimators discussed by several authors are implemented through the AMSEs. From these considerations, together with the simulation results, the advantages of our weighting scheme are reported.
\end{abstract}

KEYWORDS: Binomial regression, Kernel, MSE, Weighting

\section{Introduction}

In different fields such as pharmacology and toxicology, it is often interested in understanding the relationship between a binary response and a covariate. The formal description for the relationship is as follows. Suppose that the response $Y$ of the subject which is encoded by $Y=1$ if it exhibits the response of interest, or $Y=0$ if not, is observed at each covariate $x$. The relationship is defined by the probability of $Y=1$ conditional on $x$ with

$$
p(x)=\operatorname{Pr}(Y=1 \mid x) .
$$

Our goal is estimating the curve $p$, which has various applications to practical situations.

There are two basic approaches for estimation of $p(x)$ : the parametric approach, with the most prominent and widely used probit and logistic models for $p$ [e.g., Berkson (1944), Bliss (1934)], and the nonparametric approach. Biological mechanisms of drug action or toxicity are often so complicated that the form of the dose response curve is largely unknown, and fitting of a wrong model can then lead to large and unpredictable biases with invalid confidence intervals. In such situations, the use of nonparametric approaches becomes to be reasonable.

Overviews of nonparametric techniques for scatter-plot smoothing can be found in many literatures such as Härdle (1990), Green and Silverman (1994), Wand and Jones (1995) and 
Simonoff (1996). These include kernel methods, smoothing splines and local likelihood methods, among which local logistic regression [Fan et al.(1995)] is specifically intended for binary response variable. Nonparametric approaches to binary data were considered by Copas (1983), Staniswalis and Cooper (1988) and Müller and Schmit (1988). Copas (1983) first suggested the use of Nadaraya-Watson estimator [Nadaraya (1964), Watson (1964)] in the binary setting. Various parametric and nonparametric techniques for estimation of the functional of $p$ have been reported in Müller and Schmit (1988) and Staniswalis and Cooper (1988) in conjunction with the practical applications. In this paper, we propose two kernel-based estimators for binomial regression. Our estimators are motivated by the kernel-based estimator such as Nadaraya-Watson type suggested and discussed by Staniswalis and Cooper (1988), and are obtained by smoothing the observed proportions at each covariate through the use of the kernel weighted by the inverse of variance of the observed proportion at each covariate.

In the next section, we mention about some estimators discussed by several authors, and then the proposed weighting scheme is presented. An ideal estimator is derived from this weighting scheme. we propose two estimators depending on the variance estimators of the observed proportions, which are practicable versions of the ideal estimator. One is obtained by using the variance estimator built up by the Berkson-type estimator of $p(x)$ at each covariate $x$, and the other is constituted through a Bayes estimator of $p(x)$ at each covariate $x$. In Section 3, theoretical performances of estimators are investigated. First, those are investigated under near exact situation, which means the case where the smoothing parameter included in estimators and the number of covariates are fixed. Second, we develop asymptotic theories under the case in which the smoothing parameter goes to zero and the sample size grows. It is corresponding to the situation familiar in nonparametric smoothing area. Formulas of the Asymptotic Mean Squared Errors (AMSEs) of estimators are presented. Bias-adjustment of the proposed estimators are also discussed. By using the formulas of AMSEs in Section 3, theoretical comparisons of estimators of $p(x)$ are implemented to understand the behavior of estimators of $p(x)$ in the small number of covariates in section 4 . The results of some exact performances through numerical comparisons are reported in Section 5. These comparisons reveal that one of proposed estimators behave well especially at the region where the probability of the interested response is low, and the other is also good for the middle range of such probability. Some considerations are discussed shortly in Section 6. Section 7 gives concluding remarks in conjunction with our future works. Outline proofs of theoretical results are presented in the Appendix.

\section{Weighted kernel estimators}

We consider the equispaced fixed design. Without lack of generality, the covariates $x_{i}$ are assumed to be $x_{i}=(i-1) /(K-1), i=1, \ldots, K$. Let $Y_{i j}$ denote the $j$-th response in $N_{i}$ subjects at the covariate $x_{i}, i=1, \ldots, K$, and the responses $Y_{i j}$ 's are mutually independent. Then $Y_{i}=\sum_{j=1}^{N_{i}} Y_{i j}$ has the binomial distribution $B\left(N_{i}, p_{i}\right)$ with parameter $p_{i}=p\left(x_{i}\right)$, and it is well known that the maximum likelihood estimator (MLE) of $p_{i}$ is given by $\bar{Y}_{i}=Y_{i} / N_{i}$ for each $i$. The data $\left(x_{i}, \bar{Y}_{i}\right), i=1, \ldots, K$, immediately motivates us to construct the polygonal 
line as one of the simplest nonparametric estimators of the curve $p(x)$, which is defined by

$$
\hat{p}_{L}(x)=(K-1)\left\{\left(x-\frac{i-2}{K-1}\right) \bar{Y}_{i}+\left(\frac{i-1}{K-1}-x\right) \bar{Y}_{i-1}\right\}, \quad x_{i-1} \leq x \leq x_{i} .
$$

where $x_{0}$ is equal to 0 . This polygonal line is a naive estimator of $p(x)$ since it is built up by just connecting data $\left(x_{i}, \bar{Y}_{i}\right), i=1, \ldots, K$ by straight lines, but it is often used in the practical purposes. When the target curve $p(x)$ is smooth, however, the polygonal line is so crude that it will not be appropriate for estimating $p(x)$.

The Nadaraya-Watson estimator is well known as a basic kernel regression estimator, which is written in this situation as

$$
\hat{p}_{N W}(x ; h)=\frac{\sum_{i=1}^{K} w_{i} Y_{i}}{\sum_{i=1}^{K} w_{i} N_{i}}
$$

[cf. Lloyd (1999)], where $w_{i}=h^{-1} \phi\left(h^{-1}\left(x_{i}-x\right)\right), h$ is the bandwidth, and throughout of this paper, a unimodal density function symmetric around the origin is adopted as a kernel $\phi$.

In addition, the Nadaraya-Watson estimator of $p(x)$ based on the data $\left(x_{i}, \bar{Y}_{i}\right), i=1, \ldots, K$ is given by

$$
\hat{p}_{M}(x ; h)=\frac{\sum_{i=1}^{K} w_{i} \bar{Y}_{i}}{\sum_{i=1}^{K} w_{i}}
$$

which was proposed in Müller (1985), and Staniswalis and Cooper (1988) utilized this estimator in multivariate covariate setting. Note that $\hat{p}_{M}$ is equal to $\hat{p}_{N W}$ if $N_{i}$ 's are equal. From the view point of the local polynomial smoothing [Ruppert and Wand (1994)], $\hat{p}_{N W}$ is characterized as the minimizer of

$$
\sum_{i=1}^{K} w_{i} \sum_{j=1}^{N_{i}}\left(Y_{i j}-\beta\right)^{2}
$$

with respect to $\beta$. Similarly, $\hat{p}_{M}$ is characterized as the minimizer of

$$
\sum_{i=1}^{K} w_{i}\left(\bar{Y}_{i}-\beta\right)^{2}
$$

with respect to $\beta$. In both of (2.1) and (2.2), the differences of the variations of the responses $Y_{i j}$ are not considered in the weights $w_{i}$. However, it seems to be natural to consider that the weights should be related to the variations of the responses, since $\bar{Y}_{i}$ has the variance $v_{i} / N_{i}$ at each $x_{i}$, where $v_{i}=p_{i}\left(1-p_{i}\right)$. Our device for constructing the estimator of $p(x)$ is, therefore, to use the weighted kernel defined by $N_{i} w_{i} / v_{i}, i=1, \ldots, K$, which gives the criterion

$$
\sum_{i=1}^{K} \frac{N_{i} w_{i}}{v_{i}}\left(\bar{Y}_{i}-\beta\right)^{2}
$$

with respect to $\beta$. Through the minimization, we have the $i d e a l$ estimator $p^{*}(x ; h)$ as

$$
p^{*}(x ; h)=\frac{\sum_{i=1}^{K} Y_{i} w_{i} / v_{i}}{\sum_{i=1}^{K} N_{i} w_{i} / v_{i}} .
$$


The phrase 'ideal' means that $p^{*}$ contains the $v_{i}$ 's which are unknown since so are the $p_{i}$ 's. Therefore, in fact, the value $v_{i}$ must be estimated and the proposed estimators in this paper would be built up by substituting appropriate estimators $\hat{v}_{i}$ 's for the $v_{i}$ 's, respectively.

To obtain the estimator of $v_{i}$, we adopt two estimators of $p_{i}$. One is defined by

$$
\hat{p}_{1 i}=\left\{\begin{array}{cc}
1 /\left(2 N_{i}\right), & Y_{i}=0, \\
\bar{Y}_{i}, & 0<Y_{i}<N_{i} \\
\left(2 N_{i}-1\right) /\left(2 N_{i}\right), & Y_{i}=N_{i}
\end{array}\right.
$$

and the other estimator is defined by

$$
\hat{p}_{2 i}=\frac{Y_{i}+\sqrt{N_{i}} / 2}{N_{i}+\sqrt{N_{i}}} .
$$

Here, $\hat{p}_{1 i}$ is constituted based on Berkson's $2 N_{i}$ rule; see Berkson(1955) and $\hat{p}_{2 i}$ is the Bayes estimator with respect to the Beta distribution $B e\left(\sqrt{N_{i}} / 2, \sqrt{N_{i}} / 2\right)$ and is also the minimax estimator. Let $\hat{v}_{r i}=\hat{p}_{r i}\left(1-\hat{p}_{r i}\right)$ be the estimator of $v_{i}$ for each $r=1,2$. The two estimators of $p(x)$ proposed in this paper are

$$
\hat{p}_{r}(x ; h)=\frac{\sum_{i=1}^{K} Y_{i} w_{i} / \hat{v}_{r i}}{\sum_{i=1}^{K} N_{i} w_{i} / \hat{v}_{r i}}, r=1,2 .
$$

\section{Theoretical performances}

In this section we develop the theory for behavior of the proposed estimators and other estimators. In subsection 3.1, we consider their performances in the near exact situation. Subsection 3.2 includes those under the situation familiar in nonparametric smoothing.

\subsection{Near exact performances}

An important way of assessing the performance of an estimator is by its MSE. Here, we aim to evaluate MSEs under near exact situation. A motivation to consider such a situation is that we want to be clear the target of estimators. Obviously, the target is nothing but $p(x)$ for all estimators, however itis known that the consistent property is guaranteed under the situation that $h$ tends to zero as $K$ and $N_{i}$ 's go to infinity. Thus a natural question arisee: how are the estimators of $p(x)$ distributed without asymptotic manipulation? For this, we aim to calculate the expectations and the variances of estimators under the situation that $h, K$ and $N_{i}$ 's are all fixed. We shall give the MSEs of estimators. First, the MSE formulas of basic estimators $\hat{p}_{L}$, $\hat{p}_{N W}$ and $\hat{p}_{M}$ can be obtained immediately as follows:

$$
\begin{aligned}
\operatorname{MSE}\left[\hat{p}_{N W}(x ; h)\right] & =\left[\frac{\sum_{i=1}^{K} N_{i} p_{i} w_{i}}{\sum_{i=1}^{K} N_{i} w_{i}}-p(x)\right]^{2}+\frac{\sum_{i=1}^{K} N_{i} v_{i} w_{i}^{2}}{\left(\sum_{i=1}^{K} N_{i} w_{i}\right)^{2}}, \\
\operatorname{MSE}\left[\hat{p}_{M}(x ; h)\right] & =\left[\frac{\sum_{i=1}^{K} p_{i} w_{i}}{\sum_{i=1}^{K} w_{i}}-p(x)\right]^{2}+\frac{\sum_{i=1}^{K} v_{i} w_{i}^{2} / N_{i}}{\left(\sum_{i=1}^{K} w_{i}\right)^{2}}
\end{aligned}
$$




$$
\begin{aligned}
& \operatorname{MSE}\left[\hat{p}_{L}(x)\right]= {\left[(K-1)\left\{\left(x-\frac{i-2}{K-1}\right) p_{i}+\left(\frac{i-1}{K-1}-x\right) p_{i-1}\right\}-p(x)\right]^{2} } \\
&+(K-1)^{2}\left\{\left(x-\frac{i-2}{K-1}\right)^{2} \frac{v_{i}}{N_{i}}+\left(\frac{i-1}{K-1}-x\right)^{2} \frac{v_{i-1}}{N_{i-1}}\right\}, \\
& x_{i-1} \leq x \leq x_{i}, i=1, \ldots, K
\end{aligned}
$$

where $x_{0}=0$. Note that above (3.1), (3.2) and (3.3) can be derived without any asymptotic manipulations. In this sense, these are exact evaluations. On the other side, an assumption is needed to obtain MSE expressions of the proposed estimators. Here, the following assumption is prepared.

Assumption $1 \quad K$ and $h$ are fixed, and $N=\sum_{i=1}^{K} N_{i} \rightarrow \infty, N_{i} / N \rightarrow 1 / K$ for $i=1,2, \ldots, K$.

We explain the reason for preparing this assumption. In fact, there is often a case where the size of $K$ cannot be set up largely. In such a situation, it is interested in the behavior of estimators when increasing the numbers of subjects $N_{i}$ 's. The estimators other than the two proposed estimators can calculate those means, variances and mean square errors exactly as mentioned above. Assumption 1 expresses the minimum situation that makes possible to calculate those amounts of two proposed estimators. Thus, it can be said that we evaluate the performances of the proposed estimators near exact situation. Direct calculations including Taylor expansion yield the following.

Theorem 3.1 Under Assumption 1, we have

$$
\begin{aligned}
\operatorname{MSE}\left[\hat{p}_{1}(x ; h)\right]= & {\left[\frac{\sum_{i=1}^{K} N_{i} w_{i} /\left(1-p_{i}\right)}{\sum_{i=1}^{K} N_{i} w_{i} / v_{i}}-p(x)\right]^{2}+\frac{2 G_{1}}{N}\left[\frac{\sum_{i=1}^{K} N_{i} w_{i} /\left(1-p_{i}\right)}{\sum_{i=1}^{K} N_{i} w_{i} / v_{i}}-p(x)\right] } \\
& +\frac{\sum_{i=1}^{K} N_{i} w_{i}^{2} / v_{i}}{\left(\sum_{i=1}^{K} N_{i} w_{i} / v_{i}\right)^{2}}+\frac{H}{N}+o\left(\frac{1}{N}\right),
\end{aligned}
$$

where

$$
\begin{aligned}
G_{1} & =\frac{1}{B^{2}} \sum_{i \neq j} \frac{N_{j} w_{i} w_{j}}{N v_{i}^{2} v_{j}}\left\{p_{i}^{3}-p_{j}\left(1-3 v_{i}\right)\right\}-\frac{1}{B^{3}} \sum_{i, j} \frac{N_{i} N_{j} w_{i}^{2} w_{j}}{N^{2} v_{i}^{3} v_{j}}\left(p_{i}-p_{j}\right)\left(1-2 p_{i}\right)^{2} \\
H & =-\frac{2}{B^{3}} \sum_{i, j} \frac{N_{i} N_{j} w_{i}^{2} w_{j}}{N^{2} v_{i}^{2} v_{j}}\left(p_{i}-p_{j}\right)\left(1-2 p_{i}\right)+\frac{1}{B^{4}} \sum_{i, j, k} \frac{N_{i} N_{j} N_{k} w_{i}^{2} w_{j} w_{k}}{N^{3} v_{i}^{3} v_{j} v_{k}}\left(1-2 p_{i}\right)^{2}\left(p_{i}-p_{j}\right)\left(p_{i}-p_{k}\right), \\
B & =\sum_{i=1}^{K} \frac{N_{i} w_{i}}{N v_{i}}
\end{aligned}
$$

Theorem 3.2 Under Assumption 1, we have

$$
\begin{aligned}
\operatorname{MSE}\left[\hat{p}_{2}(x ; h)\right]= & {\left[\frac{\sum_{i=1}^{K} N_{i} w_{i} /\left(1-p_{i}\right)}{\sum_{i=1}^{K} N_{i} w_{i} / v_{i}}-p(x)\right]^{2}+2\left\{\frac{G_{21}}{\sqrt{N}}+\frac{G_{22}}{N}\right\}\left[\frac{\sum_{i=1}^{K} N_{i} w_{i} /\left(1-p_{i}\right)}{\sum_{i=1}^{K} N_{i} w_{i} / v_{i}}-p(x)\right] } \\
& +\frac{G_{21}^{2}}{N}+\frac{\sum_{i=1}^{K} N_{i} w_{i}^{2} / v_{i}}{\left(\sum_{i=1}^{K} N_{i} w_{i} / v_{i}\right)^{2}}+\frac{H}{N}+o\left(\frac{1}{N}\right)
\end{aligned}
$$


where

$$
\begin{aligned}
G_{21}= & -\frac{1}{2 B^{2}} \sum_{i, j} \frac{N_{j} \sqrt{N_{i}} w_{i} w_{j}}{N \sqrt{N} v_{i}^{2} v_{j}}\left(p_{i}-p_{j}\right)\left(1-2 p_{i}\right)^{2}, \\
G_{22}= & G_{1}+\frac{1}{4 B^{2}} \sum_{i, j} \frac{N_{i} w_{i} w_{j}}{N v_{i}^{3} v_{j}}\left(p_{i}-p_{j}\right)\left(1-2 p_{i}\right)^{2}\left(1-v_{i}\right) \\
& -\frac{1}{4 B^{3}} \sum_{i, j, k} \frac{N_{j} \sqrt{N_{i} N_{k}} w_{i} w_{j} w_{k}}{N^{2} v_{i}^{2} v_{j} v_{k}^{2}}\left(p_{i}-p_{j}\right)\left(1-2 p_{i}\right)^{2}\left(1-2 p_{k}\right)^{2},
\end{aligned}
$$

and $B, G_{1}$ and $H$ are presented in Theorem 3.1.

The sketch of proofs for Theorems 3.1 and 3.2 is given in the Appendix.

\subsection{Asymptotic performances}

Here, again we look at estimators discussed above as estimators of a curve $p(x)$. Then it is natural to consider the nonparametric smoothing situation such as the smoothing parameter tends to zero as sample size grows. Indeed, estimators including the bandwidth $h$ are not endowed with consistency without such assumptions. On the other hand, the formula of $\operatorname{MSE}\left[\hat{p}_{L}(x)\right]$ does not change under such asymptotic situation since there is no smoothing parameter. Necessary assumptions are as follows.

Assumption $2 h \rightarrow 0$ as $K \rightarrow \infty, N_{i}=N_{1} \rightarrow \infty$ for $i=2,3, \ldots, K$, in such a manner that $K h^{3+\varepsilon}=O(1)$ for some $0<\varepsilon<1$ and $N_{1} h^{2} \rightarrow 0$.

Assumption 3 The kernel $\phi$ has support on $[-1,1]$.

Assumption 4 The curve $p$ has continuous and bounded second order derivatives.

Note that $\hat{p}_{N W}$ equals $\hat{p}_{M}$ under these assumptions, since $N_{i}=N_{1}$ for $i=2, \ldots, K$. Under Assupmtions 2, 3 and 4 , the MSE of $\hat{p}_{N W}$ is given by

$$
\operatorname{MSE}\left[\hat{p}_{N W}(x ; h)\right]=\operatorname{AMSE}\left[\hat{p}_{N W}(x ; h)\right]+O\left(\frac{h}{K}+h^{6}\right),
$$

where

$$
\operatorname{AMSE}\left[\hat{p}_{N W}(x ; h)\right]=\frac{v(x) R(\phi)}{N_{1} K h}+\frac{h^{4} \mu_{2}(\phi)^{2}}{4} p^{\prime \prime}(x)^{2},
$$

$R(\phi)=\int_{-1}^{1} \phi(z)^{2} d z$ and $\mu_{2}(\phi)=\int_{-1}^{1} z^{2} \phi(z) d z$. By using the Taylor expansion, we obitain

$$
\operatorname{MSE}\left[p^{*}(x ; h)\right]=\operatorname{AMSE}\left[p^{*}(x ; h)\right]+O\left(\frac{h}{K}+h^{6}\right),
$$

where

$$
\operatorname{AMSE}\left[p^{*}(x ; h)\right]=\frac{v(x) R(\phi)}{N_{1} K h}+\frac{h^{4} \mu_{2}(\phi)^{2}}{4}\left\{p^{\prime \prime}(x)-\frac{2(1-2 p(x)) p^{\prime}(x)^{2}}{v(x)}\right\}^{2} .
$$

Next our focus goes to the proposed estimators. We have the following. 
Theorem 3.3 Under Assumtions 2, 3 and 4, we have

$$
\begin{gathered}
\operatorname{MSE}\left[\hat{p}_{1}(x ; h)\right]=\operatorname{AMSE}\left[\hat{p}_{1}(x ; h)\right]+O\left(\frac{h}{K}+h^{6}\right), \\
\operatorname{AMSE}\left[\hat{p}_{1}(x ; h)\right]=\operatorname{AMSE}\left[p^{*}(x ; h)\right]-\frac{h^{2}}{N_{1}}\{1-2 p(x)\} .
\end{gathered}
$$

Theorem 3.4 Under Assumptions 2, 3 and 4, we have

$$
\begin{aligned}
\operatorname{MSE}\left[\hat{p}_{2}(x ; h)\right] & =\operatorname{AMSE}\left[\hat{p}_{2}(x ; h)\right]+O\left(\frac{h}{K}+h^{6}\right), \\
\operatorname{AMSE}\left[\hat{p}_{2}(x ; h)\right] & =\operatorname{AMSE}\left[p^{*}(x ; h)\right]-\frac{h^{2}}{N_{1}}\{1-2 p(x)\} .
\end{aligned}
$$

Proofs of Theorems 3.3 and 3.4 are also given in the Appendix. As a common structure, the term $v(x) R(\phi)\left\{N_{1} K h\right\}^{-1}$ appears in all of MSE expressions above, which is the leading term of the variance of $\hat{p}_{N W}, p^{*}, \hat{p}_{1}$ and $\hat{p}_{2}$. Thus, we note that essential difference of these estimators appears in the bias. We can immediately obtain the following.

Corollary 3.5 If $(1-2 p(x))\left\{v(x) p^{\prime \prime}(x)-(1-2 p(x)) p^{\prime}(x)^{2}\right\} \geq 0$ for $x \in[0,1]$, then

$$
\operatorname{MSE}\left[p^{*}(x ; h)\right] \leq \operatorname{MSE}\left[\hat{p}_{N W}(x ; h)\right]
$$

by neglecting the terms of $O\left(h K^{-1}+h^{6}\right)$ and smaller.

Estimators $\hat{p}_{1}$ and $\hat{p}_{2}$ are constructed by plugging the variance estimator into $p^{*}$. However, Theorems 3.3 and 3.4 reveals that there are $O\left(h^{2} N_{1}^{-1}\right)$ differences in AMSE expressions of proposed estimators and ideal estimator. We can also say that the effect of the plug-in variance estimator is appeared as $O\left(h^{2} N_{1}^{-1}\right)$ in the sence of MSE. The order of $O\left(h^{2} N_{1}^{-1}\right)$ is important since it is dominating order in MSE expressions of $\hat{p}_{1}$ and $\hat{p}_{2}$ under Assumption 2. In the sequel, we discuss a manipulation that the MSE of $\hat{p}_{r}(r=1,2)$ are to be close to that of $p^{*}$. We see from the calculations presented in the Appendix that

$$
\operatorname{Bias}\left[\hat{p}_{r}(x)\right]=\frac{h^{2}}{2} \mu_{2}(\phi)\left\{p^{\prime \prime}(x)-\frac{2(1-2 p(x)) p^{\prime}(x)^{2}}{v(x)}\right\}-\frac{1-2 p(x)}{N_{1}}+o\left(h^{2}+\frac{1}{N_{1}}\right),
$$

for $r=1,2$. Hence the bias-adjusted $\hat{p}_{1}$ and $\hat{p}_{2}$ are defined respectively as

$$
\hat{p}_{3}(x)=\frac{1}{N_{1}}+\left(1-\frac{2}{N_{1}}\right) \hat{p}_{1}(x)
$$

and

$$
\hat{p}_{4}(x)=\frac{1}{N_{1}}+\left(1-\frac{2}{N_{1}}\right) \hat{p}_{2}(x) .
$$

Then we have the following asymptotic results.

Theorem 3.5 Under Assumtions 2, 3 and 4, we have

$$
\operatorname{MSE}\left[\hat{p}_{3}(x ; h)\right]=\operatorname{AMSE}\left[p^{*}(x ; h)\right]+O\left(\frac{h}{K}+h^{6}\right) .
$$

Theorem 3.6 Under Assumptions 2, 3 and 4, we have

$$
\operatorname{MSE}\left[\hat{p}_{4}(x ; h)\right]=\operatorname{AMSE}\left[p^{*}(x ; h)\right]+O\left(\frac{h}{K}+h^{6}\right) \text {. }
$$




\section{Asymptotic comparisons}

\subsection{The AMSE comparison under Assumption 1}

In situations under Assumption 1, we can assess the precisions of estimators by using (3.1), $(3.2),(3.3),(3.4)$ and (3.5). We considered the case that $N_{i}, i=1, \ldots, K$ are all equal. For $K=4,5$ and $N_{i}=150,200$ and 250, we compared the AMSE of four estimators $\hat{p}_{1}, \hat{p}_{2}, \hat{p}_{N W}$ and $\hat{p}_{L}$, where each AMSE is nothing but the MSE neglecting terms of $o(1 / N) \cdot \hat{p}_{1}, \hat{p}_{2}$ and $\hat{p}_{N W}$ include bandwidths $h$, and it was selected through the minimization of the mean prediction error (MPE) for each estimator, where the MPE of an estimator $\bar{p}(x)$ of $p(x)$ is defined by $\operatorname{MPE}[\bar{p}]=\sum_{i=1}^{K} \operatorname{MSE}\left[\bar{p}\left(x_{i}\right)\right]$. We adopted the true curve $p(x)$ belonging to the logistic model

$$
p_{\operatorname{logit}}\left(x ; \beta_{1}, \beta_{2}\right)=\frac{1}{1+\exp \left(\beta_{1}-\beta_{2} x\right)},
$$

where $\beta_{1}$ and $\beta_{2}$ are unknown parameters. Among this model, $p_{\text {logit }}(x ; 4,8)$ and $p_{\text {logit }}(x ; 4,2)$ were picked up as the true curves (Figure 1 ). Note that $p_{\text {logit }}(x ; 4,8)$ is a symmetric sigmoid function and $p_{\text {logit }}(x ; 4,2)$ is a flat function which gives low probabilities of responses of objects. Such situations often appear on practical situations. The normal distribution function was used as the kernel $\phi$.

Figure 1 should be inserted around here.

Figure 2 should be inserted around here.

Figure 3 should be inserted around here.

Figures 2 and 3 show the graphs of MSE for $p(x)=p_{\text {logit }}(x ; 4,8)$ and $p_{\text {logit }}(x ; 4,2)$ against $x$, respectively, for $K=5$ and $N_{i}=200$. Table 1 and Table 2 include the optimal value of MPE for the curves $p(x)=p_{\text {logit }}(x ; 4,8)$ and $p_{\text {logit }}(x ; 4,2)$, respectively, with the optional parameters $N_{i}=150,200$ and 250 , and $K=5$.

Table 1 should be inserted around here.

Table 2 should be inserted around here.

In Figure 2, the behavior of three kernel estimators are intense except around covariates $x_{i}$ 's, which may be caused by the MPE criterion for the bandwidth choice. We can observe that the polygonal line $\hat{p}_{L}(x)$ relatively surpasses the others. In Figure 3, conversely, a polygonal line is not good. In both Tables 3 and 4 , the $\operatorname{MPE}\left[\hat{p}_{L}(x)\right]$ is the largest than others. for $N_{i}=150,200$ and 250. Almost similar results were obtained also in the case $K=4$. 


\subsection{The AMSE comparison under Assumptions 2, 3 and 4}

Next we mention about a relation between $\hat{p}_{N W}$ and $p^{*}$ found from the bias expressions under Assumptions 2, 3 and 4. The $O\left(h^{2}\right)$ bias functions of $\hat{p}_{N W}$ and $p^{*}$ are, omitting the common constants, $p^{\prime \prime}(x) / 2$ and $p^{\prime \prime}(x) / 2-(1-2 p(x)) p^{\prime}(x)^{2} / v(x)$ respectively. Under the case in which $p(x)$ is a logistic distribution function, these are equal except the sign. Comparison of AMSE of $p^{*}$ and $\hat{p}_{N W}$ is easily accomplished through the function $g(x)=$ $(1-2 p(x))\left\{v(x) p^{\prime \prime}(x)-(1-2 p(x)) p^{\prime}(x)^{2}\right\}$ documented in Corollary 3.5. Figure 5 exhibits the function $g(x)$ for the curves $p(x)=0.2 \sin (2 \pi x)+0.5$ and $0.4 \sin (2 \pi x)+0.5$ (Figure 4). Figure 5 reveal that $\operatorname{MSE}\left[p^{*}(x ; h)\right] \leq \operatorname{MSE}\left[p_{N W}(x ; h)\right]$ asymptotically holds since $g(x)$ for the former $p(x)$ is positive for any $x$. However for the latter, such uniform superiority vanishes.

Figure 4 should be inserted around here.

Figure 5 should be inserted around here.

\section{Exact performances}

For $p(x)=p_{\text {logit }}\left(x ; \beta_{1}, \beta_{2}\right)$, and several values of $K$ and $N_{i}$ which are smaller than those in subsection 4.1, we carried out the exact calculations of MSEs of estimators discussed in the previous sections. Estimators compared were $\hat{p}_{r}(r=1,2,3,4), \hat{p}_{N W}$ and the parametric plug-in estimator $\hat{p}_{\mathrm{MLE}}(x)=p_{\text {logit }}\left(x ; \hat{\beta}_{1}, \hat{\beta}_{2}\right)$, where $\hat{\beta}_{1}$ and $\hat{\beta}_{2}$ are MLEs of $\beta_{1}$ and $\beta_{2}$, respectively. Note that MLEs does not always exist uniquely in this logistic model. Thus comparative studies were implemented by the conditional MSEs of estimators of $p(x)$ given the condition that the MLE for the logistic model exists uniquely, which can be calculated exactly [see, Silvapulle (1981)]. Figure 6 and 7 show the plots of the exact MSEs of estimators for the cases $p(x)=p_{\text {logit }}(x ; 4,8)$ and $p_{\text {logit }}(x ; 4,2)$ against $x$, respectively, and for $K=5$ and $N_{i}=10$. Table 3 and Table 4 include the optimal MPE for the curves $p(x)=p_{\text {logit }}(x ; 4,8)$ and $p_{\text {logit }}(x ; 4,2)$, respectively, with the optional parameters $N_{i}=5,10$ and 15 , and $K=5$.

Figure 6 should be inserted around here.

Figure 7 should be inserted around here.

Table 3 should be inserted around here.

Table 4 should be inserted around here.

In Figure $6, \hat{p}_{2}$ performs better than other estimators. $\hat{p}_{1}$ performs well in the portion of the both ends of the range. The bias-adjusted estimators $\hat{p}_{3}$ and $\hat{p}_{4}$ improve the MSE values of $\hat{p}_{1}$ and $\hat{p}_{2}$ for $x$ around 0.5 , and these are also better than $\hat{p}_{\text {MLE }}$. However, such improvements can not be observed for $x$ near boundaries. At the boudaries, $\hat{p}_{\mathrm{MLE}}$ is the best. 
In Figure 7, we can recognize $\hat{p}_{1}$ and $\hat{p}_{2}$ behave well. Especially, in the range with low probability of the response of a subject, $\hat{p}_{2}$ is superior to other estimators. We emphasize that, in spite of model-specified case, both $\hat{p}_{1}$ and $\hat{p}_{2}$ are better than $\hat{p}_{\text {MLE }}$ uniformly in $x$, and are suprior than $\hat{p}_{N W}$ for almost $x$. This fact reveals the usefulness of proposed estimators, thus that of the proposed weighting scheme, for the binomial regression problem.

We can observe from Table 4 that $\hat{p}_{1}$ and $\hat{p}_{2}$ are superior than others. From the view of MPE, bias-adjustment does not work. It seems that huge sample is needed to the merit of bias-adjustment. In $K=4$, the almost similar results were obtained although the behavior of estimators changed partly. In these experiments, $\hat{p}_{L}$ was never better than other estimators compared in this section.

\section{Considerations}

In the previous sections, we have investigated the performances of our proposed estimators. In Section 4, the behavior of estimators under small fixed $K$ (Assumption 1) were studied. We observed that kernel-based estimators behaved not so well provided $N_{i}$ 's grew. Next we implemented the comparison of $O\left(h^{2}\right)$ bias functions of kernel-based estimators. We can confirm that ideal estimator is totally better than $\hat{p}_{N W}$ if the function $g(x) \geq 0$ for any $x \in[0,1]$. Thus we expect $\hat{p}$ with efficient estimator of $v$ also performs well. This motivates us to utilize a "good" estimator of $v$, and in fact we adopted $\hat{v}_{1}$ and $\hat{v}_{2}$. Since these include stochastic error terms, the resultant $\hat{p}_{i}$ 's are also affected by its variations, which causes the stochastic error terms figured in Theorems 3.1-3.4.

The cases $K$ and $N_{i}$ are both small, which adopted in Section 5, are important for practical purpose. Estimating $p(x)$ for which $p(x)$ itself is near 0 or 0.5 has been discussed by several authors from the practical viewpoint. Our $\hat{p}_{1}$ is recommended for the case the value of $p(x)$ is small, and $\hat{p}_{2}$ is suited for the value of $p(x)$ around 0.5 . But in fact, choice $\hat{p}_{1}$ or $\hat{p}_{2}$ is depending on the practical purpose.

On the other hand, we give some comments also from the viewpoint of asymptotic. $\hat{p}_{N W}(x)$ and $\hat{p}^{*}(x)$ are asymptotically equivalent in the sense of MSE provided $p(x)$ is near to 0.5 , which can be easily confirmed by the form of $O\left(h^{2}\right)$ bias function of $p^{*}$. And the same results can be implied for $\hat{p}_{1}(x)$ and $\hat{p}_{2}(x)$, since $O\left(N_{1}^{-1}\right)$ bias function is $1-2 p(x)$. Hence the weighting scheme itself and bias-adjustment discussed in Section 3 are asymptotically not necessary around $x$ for which $p(x)=0.5$. Note that these equivalences can be observed from Figure 2. Asymptotically important problem is therefore estimation of $p(x)$ near to 0 or 1 . Figure 6 and 7 exhibit the successful of weighting though the sample size is not so large.

\section{Concluding remarks}

Kernel-based estimators for nonparametric binomial regression have been proposed, which are built up through the weighting by variations of responses. Our weighting scheme is naturally introduced and argue that it is efficient for estimating $p(x)$ even when a certain efficient parametric model for $p(x)$ is provided.

Asymptotic distributional result, such as asymptotic normality of estimators, is not pursued in this paper. Estimating a certain range of covariate $x$ is not discussed. It is also important 
for practical problem and data-based bandwidth selection is omitted though it is crucial from the computational viewpoint. We defer all of these problems to future work.

\section{Appendix}

The proofs of Theorems 3.1, 3.2, 3.3 and 3.4 are given here. For these, we needs some lemmas which are documented in the end of the Appendix. Let $\hat{\mathbf{v}}$ denote either $\hat{\mathbf{v}}_{1}=\left(\hat{v}_{11}, \hat{v}_{12}, \ldots, \hat{v}_{1 K}\right)^{T}$, an estimator derived from the Berkson's rule, or $\hat{\mathbf{v}}_{2}=\left(\hat{v}_{21}, \hat{v}_{22}, \ldots, \hat{v}_{2 K}\right)^{T}$, the minimax estimator. Then note that the components of $\hat{\mathbf{v}}$ have the asymptotic order $O_{P}\left(1 / \sqrt{N_{i}}\right)$. And let $\hat{p}_{\hat{v}}$ denote either $\hat{p}_{1}$ or $\hat{p}_{2}$. We shall perform the calculations of $E\left[\hat{p}_{\hat{v}}(x ; h)\right]$ and $V\left[\hat{p}_{\hat{v}}(x ; h)\right]$.

Proof of Theorem 3.1 and Theorem 3.2. Under Assumption 1, Taylor expansion around $\mathbf{v}=$ $\left(v_{1}, v_{2}, \ldots, v_{K}\right)^{T}$ yields that

$$
\hat{p}_{\hat{v}}(x ; h)=p^{*}(x ; h)+\Theta_{1}^{T}\left(\mathbf{I}_{K} \otimes \overline{\mathbf{Y}}\right) \mathbf{c}+\frac{1}{2} \operatorname{Tr}\left[\Theta_{2}^{T}\left(\mathbf{I}_{K} \otimes \overline{\mathbf{Y}}\right) \mathbf{c c}^{T}\right]+O_{p}\left(\frac{1}{N \sqrt{N}}\right),
$$

where $\mathbf{c}=\hat{\mathbf{v}}-\mathbf{v}, \overline{\mathbf{Y}}=\left(\bar{Y}_{1}, \bar{Y}_{2}, \ldots, \bar{Y}_{K}\right)^{T}, \mathbf{I}_{K}$ is the $K \times K$ identity matrix and $\otimes$ designates the Kronecker product. $\Theta_{1}=\left(\theta_{11}^{T}, \theta_{12}^{T}, \ldots, \theta_{1 K}^{T}\right)^{T}$ is the partitioned $K^{2} \times 1$ vector whose partitioned $i$-th $K$-dimensional column subvector is given by

$$
\frac{N_{i} w_{i}}{N B(\mathbf{v})^{2} v_{i}^{2}}\left(\mathbf{d}-B(\mathbf{v}) \mathbf{e}_{i}\right), i=1, \ldots, K
$$

where $\mathbf{d}=\left(N_{1} w_{1} /\left\{N v_{1}\right\}, N_{2} w_{2} /\left\{N v_{2}\right\}, \ldots, N_{K} w_{K} /\left\{N v_{K}\right\}\right)^{T}, B=B(\mathbf{v})=\mathbf{1}^{T} \mathbf{d}, \mathbf{e}_{i}$ is the $K$ dimensional column vector whose entries are all zero except $i$-th entry 1 . $\Theta_{2}=\left[\Theta_{21}^{T}, \Theta_{22}^{T}, \ldots, \Theta_{2 K}^{T}\right]^{T}$ is the partitioned $K^{2} \times K$ matrix, where $\Theta_{2 i}$ is the $K \times K$ matrix whose $j$-th column is the $K$-dimensional column vector given by

$$
\begin{cases}\frac{N_{i} N_{j} w_{i} w_{j}}{N^{2} v_{i}^{2} v_{j}^{2} B(\mathbf{v})^{3}}\left\{2 \mathbf{d}-B(\mathbf{v})\left(\mathbf{e}_{i}+\mathbf{e}_{j}\right)\right\}, & i \neq j \\ \frac{2 N_{i} w_{i}}{N v_{i}^{3} B(\mathbf{v})^{3}} \mathbf{e}_{i}^{T}\left(\mathbf{d}-B(\mathbf{v}) \mathbf{e}_{i}\right)\left(\mathbf{d}-B(\mathbf{v}) \mathbf{e}_{i}\right), & i=j\end{cases}
$$

We note that all components of $\Theta_{1}$ and $\Theta_{2}$ are $O(1)$ under Assumption 1. Then it follows that

$$
\begin{aligned}
E\left[\hat{p}_{\hat{v}}(x ; h)\right]=E\left[p^{*}(x ; h)\right]+\Theta_{1}^{T} E\left[\left(\mathbf{I}_{K} \otimes \overline{\mathbf{Y}}\right) \mathbf{c}\right] & \\
& +\frac{1}{2} \operatorname{Tr}\left[\Theta_{2}^{T} E\left[\left(\mathbf{I}_{K} \otimes \overline{\mathbf{Y}}\right) \mathbf{c c}^{T}\right]\right]+o\left(\frac{1}{N}\right)
\end{aligned}
$$

and

$$
\begin{aligned}
V\left[\hat{p}_{\hat{v}}(x ; h)\right]=V\left[p^{*}(x ; h)\right]+\Theta_{1}^{T} V\left[\left(\mathbf{I}_{K} \otimes \overline{\mathbf{Y}}\right) \mathbf{c}\right] \Theta_{1} \\
+2 \operatorname{Cov}\left[p^{*}(x ; h), \Theta_{1}^{T}\left(\mathbf{I}_{K} \otimes \overline{\mathbf{Y}}\right) \mathbf{c}\right]+o\left(\frac{1}{N}\right) .
\end{aligned}
$$


Let us denote the components of $\theta_{1 i}$ as $\theta_{1 i, j}$ for $i, j=1,2, \ldots, K$. Direct calculations give that

$$
\Theta_{1}^{T} E\left[\left(\mathbf{I}_{K} \otimes \overline{\mathbf{Y}}\right) \mathbf{c}\right]=\sum_{i=1}^{K} \sum_{j=1}^{K} \theta_{1 i, j} E\left[c_{i} \bar{Y}_{j}\right]
$$

Since

$$
\begin{aligned}
& E\left[c_{i} \bar{Y}_{i}\right]=\frac{1}{N_{i}} v_{i}\left(1-3 p_{i}\right)+o\left(\frac{1}{N_{i}}\right), \\
& E\left[c_{i} \bar{Y}_{j}\right]=-\frac{p_{j} v_{i}}{N_{i}}+o\left(\frac{1}{N_{i}}\right)(i \neq j),
\end{aligned}
$$

for $\hat{p}_{1}$ and

$$
\begin{aligned}
& E\left[c_{i} \bar{Y}_{i}\right]=\frac{p_{i}}{2 \sqrt{N_{i}}}\left(1-2 p_{i}\right)^{2}+\frac{p_{i}}{4 N_{i}}\left(1-4 p_{i}\right)+o\left(\frac{1}{N_{i}}\right) \\
& E\left[c_{i} \bar{Y}_{j}\right]=\frac{p_{j}}{2 \sqrt{N_{i}}}\left(1-2 p_{i}\right)^{2}+\frac{p_{j}}{4 N_{i}}\left(8 v_{i}-3\right)+o\left(\frac{1}{N_{i}}\right)(i \neq j)
\end{aligned}
$$

for $\hat{p}_{2}$, we have

$$
\Theta_{1}^{T} E\left[\left(\mathbf{I}_{K} \otimes \overline{\mathbf{Y}}\right) \mathbf{c}\right]=\frac{1}{B(\mathbf{v})^{2}} \sum_{i \neq j} \frac{N_{j} w_{i} w_{j}}{N^{2} v_{i} v_{j}}\left(3 p_{i}-p_{j}-1\right)+o\left(\frac{1}{N}\right),
$$

for $\hat{p}_{1}$ and

$$
\begin{aligned}
\Theta_{1}^{T} E\left[\left(\mathbf{I}_{K} \otimes \overline{\mathbf{Y}}\right) \mathbf{c}\right]= & -\frac{1}{2 B(\mathbf{v})^{2}} \sum_{i, j} \frac{N_{j} \sqrt{N_{i}} w_{i} w_{j}}{N^{2} v_{i}^{2} v_{j}}\left(p_{i}-p_{j}\right)\left(1-2 p_{i}\right)^{2} \\
& +\frac{1}{4 B(\mathbf{v})^{2}} \sum_{i \neq j} \frac{N_{j} w_{i} w_{j}}{N^{2} v_{i}^{2} v_{j}}\left\{3\left(p_{i}-p_{j}\right)-4 v_{i}\left(1-2 p_{j}\right)\right\}+o\left(\frac{1}{N}\right),(\mathrm{A}
\end{aligned}
$$

for $\hat{p}_{2}$. Next we calculate $\operatorname{Tr}\left[\Theta_{2}^{T} E\left[\left(\mathbf{I}_{K} \otimes \overline{\mathbf{Y}}\right) \mathbf{c c}^{T}\right]\right]$. It follows from the properties of the Kronecker product that $\operatorname{Tr}\left[\Theta_{2}^{T} E\left[\left(\mathbf{I}_{K} \otimes \overline{\mathbf{Y}}\right) \mathbf{c} \mathbf{c}^{T}\right]\right]=\sum_{i=1}^{K} \operatorname{Tr}\left[\Theta_{2 i} E\left[c_{i} \overline{\mathbf{Y}} \mathbf{c}^{T}\right]\right]$. Denote the $(j, k)-$ component of $\Theta_{2 i}$ as $\Theta_{2 i, j k}$, and note that the $(j, k)$-component of $E\left[c_{i} \mathbf{Y} \mathbf{c}^{T}\right]$ is $E\left[c_{i} \bar{Y}_{j} c_{k}\right]$, we have

$$
\operatorname{Tr}\left[\Theta_{2}^{T} E\left[\left(\mathbf{I}_{K} \otimes \overline{\mathbf{Y}}\right) \mathbf{c c}^{T}\right]\right]=\sum_{i=1}^{K} \sum_{j=1}^{K} \sum_{k=1}^{K} \Theta_{2 i, k j} E\left[c_{i} c_{j} \bar{Y}_{k}\right] .
$$

Necesarry calculations are

$$
\begin{aligned}
E\left[c_{i} c_{j} \bar{Y}_{k}\right] & =o\left(\frac{1}{N_{i}}\right)(i \neq j, j \neq k, i \neq k), \\
E\left[c_{i}^{2} \bar{Y}_{j}\right] & =\frac{1}{N_{i}} v_{i}\left(1-2 p_{i}\right)^{2} p_{j}+o\left(\frac{1}{N_{i}}\right)(i \neq j), \\
E\left[c_{i} c_{j} \bar{Y}_{j}\right] & =o\left(\frac{1}{N_{i}}\right)(i \neq j), \\
E\left[c_{i}^{2} \bar{Y}_{i}\right] & =\frac{1}{N_{i}} v_{i}\left(1-2 p_{i}\right)^{2} p_{i}+o\left(\frac{1}{N_{i}}\right),
\end{aligned}
$$


for $\hat{p}_{1}$ and

$$
\begin{aligned}
E\left[c_{i} c_{j} \bar{Y}_{k}\right] & =\frac{1}{4 \sqrt{N_{i} N_{j}}}\left(1-2 p_{i}\right)^{2}\left(1-2 p_{j}\right)^{2} p_{k}+o\left(\frac{1}{\sqrt{N_{i} N_{j}}}\right)(i \neq j, j \neq k, i \neq k), \\
E\left[c_{i}^{2} \bar{Y}_{j}\right] & =\frac{1}{4 N_{i}}\left(1-2 p_{i}\right)^{2} p_{j}+o\left(\frac{1}{N_{i}}\right)(i \neq j), \\
E\left[c_{i} c_{j} \bar{Y}_{j}\right] & =\frac{1}{4 \sqrt{N_{i} N_{j}}}\left(1-2 p_{i}\right)^{2}\left(1-2 p_{j}\right)^{2} p_{j}+o\left(\frac{1}{\sqrt{N_{i} N_{j}}}\right)(i \neq j), \\
E\left[c_{i}^{2} \bar{Y}_{i}\right] & =\frac{1}{4 N_{i}}\left(1-2 p_{i}\right)^{2} p_{i}+o\left(\frac{1}{N_{i}}\right),
\end{aligned}
$$

for $\hat{p}_{2}$, and these are combined to give

$$
\begin{aligned}
\operatorname{Tr}\left[\Theta_{2}^{T} E\left[\left(\mathbf{I}_{K} \otimes \overline{\mathbf{Y}}\right) \mathbf{c} \mathbf{c}^{T}\right]\right]= & \frac{1}{B(\mathbf{v})^{2}} \sum_{i, j} \frac{N_{j} w_{i} w_{j}}{N^{2} v_{i}^{2} v_{j}}\left(p_{i}-p_{j}\right)\left(1-2 p_{i}\right)^{2} \\
& -\frac{1}{B(\mathbf{v})^{3}} \sum_{i, j} \frac{N_{i} N_{j} w_{i}^{2} w_{j}}{N^{3} v_{i}^{3} v_{j}}\left(p_{i}-p_{j}\right)\left(1-2 p_{i}\right)^{2}+o\left(\frac{1}{N}\right)
\end{aligned}
$$

for $\hat{p}_{1}$ and

$$
\begin{aligned}
\operatorname{Tr}\left[\Theta_{2}^{T} E\left[\left(\mathbf{I}_{K} \otimes \overline{\mathbf{Y}}\right) \mathbf{c c}^{T}\right]\right]= & \frac{1}{B(\mathbf{v})^{2}} \sum_{i, j} \frac{N_{j} w_{i} w_{j}}{4 N^{2} v_{i}^{3} v_{j}}\left(p_{i}-p_{j}\right)\left(1-2 p_{i}\right)^{2} \\
& -\frac{1}{B(\mathbf{v})^{3}}\left[\sum_{i, j} \frac{N_{i} N_{j} w_{i}^{2} w_{j}}{N^{3} v_{i}^{3} v_{j}}\left(p_{i}-p_{j}\right)\left(1-2 p_{i}\right)^{2}\right. \\
& \left.+\sum_{i, j, k} \frac{N_{j} \sqrt{N_{i} N_{k}} w_{i} w_{j} w_{k}}{4 N^{3} v_{i}^{2} v_{j} v_{k}^{2}}\left(p_{i}-p_{j}\right)\left(1-2 p_{i}\right)^{2}\left(1-2 p_{k}\right)^{2}\right] \\
& +o\left(\frac{1}{N}\right)
\end{aligned}
$$

for $\hat{p}_{2}$.Using (A.3), (A.4), (A.5) and (A.6) and with suitable arrangements, we obtain

$$
\left\{E\left[\hat{p}_{\hat{v}}(x ; h)\right]-p(x)\right\}^{2}=\left\{E\left[p^{*}(x ; h)\right]-p(x)\right\}^{2}+\frac{2 G_{1}}{N}\left\{E\left[p^{*}(x ; h)\right]-p(x)\right\}+o\left(\frac{1}{N}\right),
$$

for $\hat{p}_{1}(x ; h)$, where $G_{1}$ is given in Theorem 3.1 , and similarly,

$$
\begin{aligned}
\left\{E\left[\hat{p}_{\hat{v}}(x ; h)\right]-p(x)\right\}^{2}= & \left\{E\left[p^{*}(x ; h)\right]-p(x)\right\}^{2}+2\left\{\frac{G_{21}}{\sqrt{N}}+\frac{G_{22}}{N}\right\}\left\{E\left[p^{*}(x ; h)\right]-p(x)\right\} \\
& +\frac{G_{21}^{2}}{N}+o\left(\frac{1}{N}\right),
\end{aligned}
$$

for $\hat{p}_{2}(x ; h)$, where $G_{21}$ and $G_{22}$ are listed in Theorem 3.2. We then have the expressions of the squared bias term in Theorems 3.1 and 3.2 by the use of

$$
E\left[p^{*}(x ; h)\right]=\frac{\sum_{i=1}^{K} N_{i} w_{i} /\left(1-p_{i}\right)}{\sum_{i=1}^{K} N_{i} w_{i} / v_{i}} .
$$


Next we turn to the variance. Easy calculation gives that

$$
V\left[p^{*}(x ; h)\right]=\frac{\sum_{i=1}^{K} N_{i} w_{i}^{2} / v_{i}}{\left(\sum_{i=1}^{K} N_{i} w_{i} / v_{i}\right)^{2}}
$$

We see from

$$
\Theta_{1}^{T} V\left[\left(\mathbf{I}_{K} \otimes \overline{\mathbf{Y}}\right) \mathbf{c}\right] \Theta_{1}=\sum_{i=1}^{K} \sum_{j=1}^{K} \sum_{k=1}^{K} \sum_{\ell=1}^{K} \theta_{1 i, j} \theta_{1 k, \ell} \operatorname{Cov}\left[c_{i} \bar{Y}_{j}, c_{k} \bar{Y}_{\ell}\right]
$$

the quantities necessarry to proceed the calculations of $\Theta_{1}^{T} V\left[\left(\mathbf{I}_{K} \otimes \overline{\mathbf{Y}}\right) \mathbf{c}\right] \Theta_{1}$ are

$$
\begin{aligned}
\operatorname{Cov}\left[c_{i} \bar{Y}_{i}, c_{i} \bar{Y}_{i}\right] & =\frac{1}{N_{i}} v_{i} p_{i}^{2}\left(1-2 p_{i}\right)^{2}+o\left(\frac{1}{N_{i}}\right), \\
\operatorname{Cov}\left[c_{i} \bar{Y}_{i}, c_{i} \bar{Y}_{j}\right] & =\frac{1}{N_{i}} v_{i} p_{i} p_{j}\left(1-2 p_{i}\right)^{2}+o\left(\frac{1}{N_{i}}\right)(i \neq j), \\
\operatorname{Cov}\left[c_{i} \bar{Y}_{i}, c_{j} \bar{Y}_{i}\right] & =o\left(\frac{1}{N_{i}}\right)(i \neq j), \\
\operatorname{Cov}\left[c_{i} \bar{Y}_{i}, c_{j} \bar{Y}_{j}\right] & =0(i \neq j), \\
\operatorname{Cov}\left[c_{i} \bar{Y}_{i}, c_{j} \bar{Y}_{k}\right] & =0(i \neq j, j \neq k, i \neq k), \\
\operatorname{Cov}\left[c_{i} \bar{Y}_{i}, c_{i} \bar{Y}_{j}\right] & =o\left(\frac{1}{N_{j}}\right)(i \neq j), \\
\operatorname{Cov}\left[c_{i} \bar{Y}_{j}, c_{i} \bar{Y}_{k}\right] & =\frac{1}{N_{i}} v_{i} p_{j} p_{k}\left(1-2 p_{i}\right)^{2}+o\left(\frac{1}{N_{i}}\right)(i \neq j, j \neq k, i \neq k), \\
\operatorname{Cov}\left[c_{i} \bar{Y}_{j}, c_{j} \bar{Y}_{i}\right] & =o\left(\frac{1}{\sqrt{N_{i} N_{j}}}\right), \\
\operatorname{Cov}\left[c_{i} \bar{Y}_{j}, c_{j} \bar{Y}_{k}\right] & =o\left(\frac{1}{N_{j}}\right)(i \neq j), \\
\operatorname{Cov}\left[c_{i} \bar{Y}_{j}, c_{k} \bar{Y}_{k}\right] & =0(i \neq j, j \neq k, i \neq k),
\end{aligned}
$$

for both $\hat{p}_{1}$ and $\hat{p}_{2}$. These are combined to give

$$
\Theta_{1}^{T} V\left[\left(\mathbf{I}_{K} \otimes \overline{\mathbf{Y}}\right) \mathbf{c}\right] \Theta_{1}=\frac{1}{B(\mathbf{v})^{4}} \sum_{i, j, k} \frac{N_{i} N_{j} N_{k} w_{i}^{2} w_{j} w_{k}}{N^{4} v_{i}^{3} v_{j} v_{k}}\left(1-2 p_{i}\right)^{2}\left(p_{i}-p_{j}\right)\left(p_{i}-p_{k}\right)+o\left(\frac{1}{N}\right)
$$

for $\hat{p}_{1}$ and $\hat{p}_{2}$. Finary we aim to evaluate $\operatorname{Cov}\left[p^{*}(x ; h), \Theta_{1}^{T}\left(\mathbf{I}_{K} \otimes \overline{\mathbf{Y}}\right) \mathbf{c}\right]$, which can be written as

$$
\operatorname{Cov}\left[p^{*}(x ; h), \Theta_{1}^{T}\left(\mathbf{I}_{K} \otimes \overline{\mathbf{Y}}\right) \mathbf{c}\right]=\frac{1}{B(\mathbf{v})} \sum_{i=1}^{K} \sum_{j=1}^{K} \sum_{k=1}^{K} \frac{N_{i} w_{i}}{N v_{i}} \theta_{1 j, k} \operatorname{Cov}\left[\bar{Y}_{i}, c_{j} \bar{Y}_{k}\right]
$$

We could calculate

$$
\operatorname{Cov}\left[\bar{Y}_{i}, c_{j} \bar{Y}_{k}\right]=0,(i \neq j, j \neq k, i \neq k)
$$




$$
\begin{aligned}
\operatorname{Cov}\left[\bar{Y}_{i}, c_{i} \bar{Y}_{j}\right] & =\frac{1}{N_{i}} v_{i} p_{j}\left(1-2 p_{i}\right)+o\left(\frac{1}{N_{j}}\right)(i \neq j), \\
\operatorname{Cov}\left[\bar{Y}_{i}, c_{j} \bar{Y}_{i}\right] & =o\left(\frac{1}{N_{j}}\right)(i \neq j), \\
\operatorname{Cov}\left[\bar{Y}_{i}, c_{i} \bar{Y}_{i}\right] & =\frac{1}{N_{i}} v_{i} p_{i}\left(1-2 p_{i}\right)+o\left(\frac{1}{N_{j}}\right)
\end{aligned}
$$

for $\hat{p}_{1}$ and $\hat{p}_{2}$. Thus we get

$$
\operatorname{Cov}\left[p^{*}(x ; h), \Theta_{1}^{T}\left(\mathbf{I}_{K} \otimes \overline{\mathbf{Y}}\right) \mathbf{c}\right]=-\frac{1}{B(\mathbf{v})^{3}} \sum_{i, j} \frac{N_{i} N_{j} w_{i}^{2} w_{j}}{N^{3} v_{i}^{2} v_{j}}\left(p_{i}-p_{j}\right)\left(1-2 p_{i}\right)+o\left(\frac{1}{N}\right)
$$

for $\hat{p}_{1}$ and $\hat{p}_{2}$. Above variance and covariance expressions are successfully combined to derive $H$ and negligible order, which completes the proof of Theorems 3.1 and 3.2.

Proof of Theorem 3.3 and Theorem 3.4. We consider the case under Assumptions 2, 3 and 4. In the first stage, our focus goes to $p^{*}$, since the performance of $\hat{p}_{\hat{v}}$ is dominated by that of $p^{*}$. Using the notations similar to those utilized in the local polynomial smoothing [Ruppert and Wand (1994)], $p^{*}(x ; h)$ can be written as $p^{*}(x ; h)=\left(\mathbf{1}^{T} W_{x} \mathbf{1}\right)^{-1} \mathbf{1}^{T} W_{x} \overline{\mathbf{Y}}$, where $W_{x}=$ $\operatorname{diag}\left\{w_{1} / v_{1}, \ldots, w_{K} / v_{K}\right\}$. Then (A.3), (A.4) and Taylor expansions under Assumptions 2, 3 and 4 , applications of Lemma 4 yield

$$
\begin{aligned}
& E\left[p^{*}(x ; h)\right]=p(x)+\frac{h^{2} \mu_{2}(\phi)}{2}\left\{p^{\prime \prime}(x)-\frac{2(1-2 p(x)) p^{\prime}(x)^{2}}{v(x)}\right\}+O\left(h^{4}+\frac{1}{K h}\right), \\
& V\left[p^{*}(x ; h)\right]=\frac{v(x) R(\phi)}{N_{1} K h}+O\left(\frac{h}{N_{1} K}+\frac{1}{N_{1} K^{2} h^{2}}\right) .
\end{aligned}
$$

Hence we must show that the extra terms in (A.7) and (A.8) are negligible order under Assumptions 2, 3 and 4. Using Lemma 4 and Taylor expansions under Assumptions 2, 3 and 4, the first term in the right side of (A.3) is

$$
\begin{aligned}
& \frac{1}{N_{1} B(\mathbf{v})^{2}} \sum_{i \neq j} \frac{w_{i} w_{j}}{K^{2} v_{i} v_{j}}\left(3 p_{i}-p_{j}-1\right) \\
= & \frac{1}{N_{1} B(\mathbf{v})^{2}}\left[\sum_{i, j} \frac{w_{i} w_{j}}{K^{2} v_{i} v_{j}}\left(3 p_{i}-p_{j}-1\right)-\sum_{i=1}^{K} \frac{w_{i}^{2}}{K^{2} v_{i}^{2}}\left(2 p_{i}-1\right)\right] \\
= & \frac{1}{N_{1} B(\mathbf{v})^{2}}\left[\left(\frac{1}{K h} \sum_{i=1}^{K} \frac{3 p\left(x_{i}\right)}{v\left(x_{i}\right)} \phi\left(\frac{x_{i}-x}{h}\right)\right)\left(\frac{1}{K h} \sum_{j=1}^{K} \frac{1}{v\left(x_{j}\right)} \phi\left(\frac{x_{j}-x}{h}\right)\right)\right. \\
& -\left(\frac{1}{K h} \sum_{i=1}^{K} \frac{1}{v\left(x_{i}\right)} \phi\left(\frac{x_{i}-x}{h}\right)\right)\left(\frac{1}{K h} \sum_{j=1}^{K} \frac{p\left(x_{j}\right)+1}{v\left(x_{j}\right)} \phi\left(\frac{x_{j}-x}{h}\right)\right) \\
& \left.-\frac{1}{K^{2} h^{2}} \sum_{i=1}^{K} \frac{2 p\left(x_{i}\right)-1}{v\left(x_{i}\right)^{2}} \phi\left(\frac{x_{i}-x}{h}\right)^{2}\right] \\
= & -\frac{1-2 p(x)}{N_{1}}+o\left(\frac{1}{N_{1}}\right),
\end{aligned}
$$


from which it follows that

$$
\Theta_{1}^{T} E\left[\left(\mathbf{I}_{K} \otimes \overline{\mathbf{Y}}\right) \mathbf{c}\right]=-\frac{1-2 p(x)}{N_{1}}+o\left(\frac{1}{N_{1}}\right)
$$

for $\hat{p}_{1}$. Similarly, the amount to the second term in the right side of (A.4) is

$$
\begin{aligned}
- & \frac{1}{2 \sqrt{N_{1}} B(\mathbf{v})^{2}} \sum_{i, j} \frac{w_{i} w_{j}}{K^{2} v_{i}^{2} v_{j}}\left(p_{i}-p_{j}\right)\left(1-2 p_{i}\right)^{2} \\
& +\frac{1}{4 N_{1} B(\mathbf{v})^{2}} \sum_{i \neq j} \frac{w_{i} w_{j}}{K^{2} v_{i}^{2} v_{j}}\left\{3\left(p_{i}-p_{j}\right)-4 v_{i}\left(1-2 p_{j}\right)\right\} \\
= & -\frac{1}{2 \sqrt{N_{1}} B(\mathbf{v})^{2}} \sum_{i, j} \frac{w_{i} w_{j}}{K^{2} v_{i}^{2} v_{j}}\left(p_{i}-p_{j}\right)\left(1-2 p_{i}\right)^{2} \\
+ & \frac{1}{4 N_{1} B(\mathbf{v})^{2}}\left[\sum_{i, j} \frac{w_{i} w_{j}}{K^{2} v_{i}^{2} v_{j}}\left\{3 p_{i}-4 v_{i}-p_{j}\left(3-8 v_{i}\right)\right\}+\sum_{i=1}^{K} \frac{4 w_{i}^{2}}{K^{2} v_{i}^{2}}\left(1-2 p_{i}\right)\right] \\
= & -\frac{1}{2 \sqrt{N_{1}} B(\mathbf{v})^{2}}\left[\left(\frac{1}{K h} \sum_{i=1}^{K} \frac{p\left(x_{i}\right)\left(1-2 p\left(x_{i}\right)\right)^{2}}{v\left(x_{i}\right)^{2}} \phi\left(\frac{x_{i}-x}{h}\right)\right)\left(\frac{1}{K h} \sum_{j=1}^{K} \frac{1}{v\left(x_{j}\right)} \phi\left(\frac{x_{j}-x}{h}\right)\right)\right. \\
& \left.-\left(\frac{1}{K h} \sum_{i=1}^{K} \frac{\left(1-2 p\left(x_{i}\right)\right)^{2}}{v\left(x_{i}\right)^{2}} \phi\left(\frac{x_{i}-x}{h}\right)\right)\left(\frac{1}{K h} \sum_{j=1}^{K} \frac{p\left(x_{j}\right)}{v\left(x_{j}\right)} \phi\left(\frac{x_{j}-x}{h}\right)\right)\right] \\
& +\frac{1}{4 N_{1} B(\mathbf{v})^{2}}\left[\left(\frac{1}{K h} \sum_{i=1}^{K} \frac{3 p\left(x_{i}\right)-4 v\left(x_{i}\right)}{v\left(x_{i}\right)^{2}} \phi\left(\frac{x_{i}-x}{h}\right)\right)\left(\frac{1}{K h} \sum_{j=1}^{K} \frac{1}{v\left(x_{j}\right)} \phi\left(\frac{x_{j}-x}{h}\right)\right)\right. \\
= & -\frac{1-2 p(x)}{N_{1}}+O\left(\frac{1}{h^{2}} \sum_{i=1}^{K} \frac{3-8 v\left(x_{i}\right)}{v\left(x_{i}\right)} \phi\left(\frac{x_{i}-x}{h}\right)\right)\left(\frac{1}{K h} \sum_{j=1}^{K} \frac{p\left(x_{j}\right)}{v\left(x_{j}\right)} \phi\left(\frac{x_{j}-x}{h}\right)\right) \\
& \left.+\frac{1}{K^{2} h^{2}} \sum_{i=1}^{K} \frac{4\left(1-2 p\left(x_{i}\right)\right)}{v\left(x_{i}\right)^{2}} \phi\left(\frac{x_{i}-x}{h}\right)^{2}\right] \\
& \left(\frac{1}{h h}\right)
\end{aligned}
$$

Hence

$$
\Theta_{1}^{T} E\left[\left(\mathbf{I}_{K} \otimes \overline{\mathbf{Y}}\right) \mathbf{c}\right]=-\frac{1-2 p(x)}{N_{1}}+O\left(\frac{h^{2}}{\sqrt{N_{1}}}\right)
$$

for $\hat{p}_{2}$. The amount to the second term in the right side of (A.5) is

$$
\begin{aligned}
& \frac{1}{N_{1} B(\mathbf{v})^{2}} \sum_{i, j} \frac{w_{i} w_{j}}{K^{2} v_{i}^{2} v_{j}}\left(p_{i}-p_{j}\right)\left(1-2 p_{i}\right)^{2} \\
& \quad-\frac{1}{N_{1} B(\mathbf{v})^{3}} \sum_{i, j} \frac{w_{i}^{2} w_{j}}{K^{2} v_{i}^{3} v_{j}}\left(p_{i}-p_{j}\right)\left(1-2 p_{i}\right)^{2} \\
& =\frac{1}{N_{1} B(\mathbf{v})^{2}}\left[\left(\frac{1}{K h} \sum_{i=1}^{K} \frac{p\left(x_{i}\right)\left(1-2 p\left(x_{i}\right)\right)^{2}}{v\left(x_{i}\right)^{2}} \phi\left(\frac{x_{i}-x}{h}\right)\right)\left(\frac{1}{K h} \sum_{j=1}^{K} \frac{1}{v\left(x_{j}\right)} \phi\left(\frac{x_{j}-x}{h}\right)\right)\right.
\end{aligned}
$$




$$
\begin{aligned}
& \left.-\left(\frac{1}{K h} \sum_{i=1}^{K} \frac{\left(1-2 p\left(x_{i}\right)\right)^{2}}{v\left(x_{i}\right)^{2}} \phi\left(\frac{x_{i}-x}{h}\right)\right)\left(\frac{1}{K h} \sum_{j=1}^{K} \frac{p\left(x_{j}\right)}{v\left(x_{j}\right)} \phi\left(\frac{x_{j}-x}{h}\right)\right)\right] \\
- & \frac{1}{N_{1} K h B(\mathbf{v})^{3}}\left[\left(\frac{1}{K h} \sum_{i=1}^{K} \frac{p\left(x_{i}\right)\left(1-2 p\left(x_{i}\right)\right)^{2}}{v\left(x_{i}\right)^{3}} \phi\left(\frac{x_{i}-x}{h}\right)^{2}\right)\left(\frac{1}{K h} \sum_{j=1}^{K} \frac{1}{v\left(x_{j}\right)} \phi\left(\frac{x_{j}-x}{h}\right)\right)\right. \\
& \left.-\left(\frac{1}{K h} \sum_{i=1}^{K} \frac{\left(1-2 p\left(x_{i}\right)\right)^{2}}{v\left(x_{i}\right)^{3}} \phi\left(\frac{x_{i}-x}{h}\right)^{2}\right)\left(\frac{1}{K h} \sum_{j=1}^{K} \frac{p\left(x_{j}\right)}{v\left(x_{j}\right)} \phi\left(\frac{x_{j}-x}{h}\right)\right)\right] \\
= & O\left(\frac{h^{2}}{N_{1}}\right) .
\end{aligned}
$$

It is easy to see that the amount to the second term in the right side of (A.5) also appears in (A.6). Necessary evaluation for (A.6) is therefore,

$$
\begin{aligned}
& \frac{1}{4 N_{1} B(\mathbf{v})^{3}} \sum_{i, j, k} \frac{w_{i} w_{j} w_{k}}{K^{3} v_{i}^{2} v_{j} v_{k}^{2}}\left(p_{i}-p_{j}\right)\left(1-2 p_{i}\right)^{2}\left(1-2 p_{k}\right)^{2} \\
& =\frac{1}{4 N_{1} B(\mathbf{v})^{3}} \\
& \times\left[\left(\frac{1}{K h} \sum_{i=1}^{K} \frac{p\left(x_{i}\right)\left(1-2 p\left(x_{i}\right)\right)^{2}}{v\left(x_{i}\right)^{2}} \phi\left(\frac{x_{i}-x}{h}\right)\right)\left(\frac{1}{K h} \sum_{j=1}^{K} \frac{1}{v\left(x_{j}\right)} \phi\left(\frac{x_{j}-x}{h}\right)\right)\right. \\
& \quad \times\left(\frac{1}{K h} \sum_{i=k}^{K} \frac{\left(1-2 p\left(x_{k}\right)\right)^{2}}{v\left(x_{k}\right)^{2}} \phi\left(\frac{x_{k}-x}{h}\right)\right) \\
& \left.-\quad \frac{1}{K h} \sum_{i=1}^{K} \frac{\left(1-2 p\left(x_{i}\right)\right)^{2}}{v\left(x_{i}\right)^{2}} \phi\left(\frac{x_{i}-x}{h}\right)\right)\left(\frac{1}{K h} \sum_{j=1}^{K} \frac{p\left(x_{j}\right)}{v\left(x_{j}\right)} \phi\left(\frac{x_{j}-x}{h}\right)\right) \\
& \left.\quad \times\left(\frac{1}{K h} \sum_{i=k}^{K} \frac{\left(1-2 p\left(x_{k}\right)\right)^{2}}{v\left(x_{k}\right)^{2}} \phi\left(\frac{x_{k}-x}{h}\right)\right)\right] \\
& =O\left(\frac{h^{2}}{N_{1}}\right),
\end{aligned}
$$

it follows that

$$
\operatorname{Tr}\left[\Theta_{2}^{T} E\left[\left(\mathbf{I}_{K} \otimes \overline{\mathbf{Y}}\right) \mathbf{c c}^{T}\right]\right]=O\left(\frac{h^{2}}{N_{1}}\right)
$$

for both $\hat{p}_{1}$ and $\hat{p}_{2}$. Further the first term in (A.9) is

$$
\begin{aligned}
& \frac{1}{N_{1} B(\mathbf{v})^{4}} \sum_{i, j, k} \frac{w_{i}^{2} w_{j} w_{k}}{K^{4} v_{i}^{3} v_{j} v_{k}}\left(1-2 p_{i}\right)^{2}\left(p_{i}-p_{j}\right)\left(p_{i}-p_{k}\right) \\
& =\frac{1}{N_{1} B(\mathbf{v})^{4}} \\
& \times\left[\left(\frac{1}{K h} \sum_{i=1}^{K} \frac{p\left(x_{i}\right)^{2}\left(1-2 p\left(x_{i}\right)\right)^{2}}{v\left(x_{i}\right)^{3}} \phi\left(\frac{x_{i}-x}{h}\right)^{2}\right)\left(\frac{1}{K h} \sum_{j=1}^{K} \frac{1}{v\left(x_{j}\right)} \phi\left(\frac{x_{j}-x}{h}\right)\right)\left(\frac{1}{K h} \sum_{i=k}^{K} \frac{1}{v\left(x_{k}\right)} \phi\left(\frac{x_{k}-x}{h}\right)\right)\right.
\end{aligned}
$$




$$
\begin{aligned}
& -\left(\frac{1}{K h} \sum_{i=1}^{K} \frac{p\left(x_{i}\right)\left(1-2 p\left(x_{i}\right)\right)^{2}}{v\left(x_{i}\right)^{3}} \phi\left(\frac{x_{i}-x}{h}\right)^{2}\right)\left(\frac{1}{K h} \sum_{j=1}^{K} \frac{p\left(x_{j}\right)}{v\left(x_{j}\right)} \phi\left(\frac{x_{j}-x}{h}\right)\right)\left(\frac{1}{K h} \sum_{i=k}^{K} \frac{1}{v\left(x_{k}\right)} \phi\left(\frac{x_{k}-x}{h}\right)\right) \\
& -\left(\frac{1}{K h} \sum_{i=1}^{K} \frac{p\left(x_{i}\right)\left(1-2 p\left(x_{i}\right)\right)^{2}}{v\left(x_{i}\right)^{3}} \phi\left(\frac{x_{i}-x}{h}\right)^{2}\right)\left(\frac{1}{K h} \sum_{j=1}^{K} \frac{1}{v\left(x_{j}\right)} \phi\left(\frac{x_{j}-x}{h}\right)\right)\left(\frac{1}{K h} \sum_{i=k}^{K} \frac{p\left(x_{k}\right)}{v\left(x_{k}\right)} \phi\left(\frac{x_{k}-x}{h}\right)\right) \\
& \left.+\left(\frac{1}{K h} \sum_{i=1}^{K} \frac{\left(1-2 p\left(x_{i}\right)\right)^{2}}{v\left(x_{i}\right)^{3}} \phi\left(\frac{x_{i}-x}{h}\right)^{2}\right)\left(\frac{1}{K h} \sum_{j=1}^{K} \frac{p\left(x_{j}\right)}{v\left(x_{j}\right)} \phi\left(\frac{x_{j}-x}{h}\right)\right)\left(\frac{1}{K h} \sum_{i=k}^{K} \frac{p\left(x_{k}\right)}{v\left(x_{k}\right)} \phi\left(\frac{x_{k}-x}{h}\right)\right)\right] \\
& =O\left(\frac{h}{N_{1} K}\right),
\end{aligned}
$$

And the first term in (A.10) is

$$
\begin{aligned}
& -\frac{1}{N_{1} B(\mathbf{v})^{3}} \sum_{i, j} \frac{w_{i}^{2} w_{j}}{K^{3} v_{i}^{2} v_{j}}\left(p_{i}-p_{j}\right)\left(1-2 p_{i}\right) \\
& =-\frac{1}{N_{1} K h B(\mathbf{v})^{3}}\left[\left(\frac{1}{K h} \sum_{i=1}^{K} \frac{p\left(x_{i}\right)\left(1-2 p\left(x_{i}\right)\right)}{v\left(x_{i}\right)^{2}} \phi\left(\frac{x_{i}-x}{h}\right)^{2}\right)\left(\frac{1}{K h} \sum_{j=1}^{K} \frac{1}{v\left(x_{j}\right)} \phi\left(\frac{x_{j}-x}{h}\right)\right)\right. \\
& \left.-\left(\frac{1}{K h} \sum_{i=1}^{K} \frac{\left(1-2 p\left(x_{i}\right)\right)}{v\left(x_{i}\right)^{2}} \phi\left(\frac{x_{i}-x}{h}\right)^{2}\right)\left(\frac{1}{K h} \sum_{j=1}^{K} \frac{p\left(x_{j}\right)}{v\left(x_{j}\right)} \phi\left(\frac{x_{j}-x}{h}\right)\right)\right] \\
& =O\left(\frac{h}{N_{1} K}\right) .
\end{aligned}
$$

Hence

$$
\Theta_{1}^{T} V\left[\left(\mathbf{I}_{K} \otimes \overline{\mathbf{Y}}\right) \mathbf{c}\right] \Theta_{1}+2 \operatorname{Cov}\left[p^{*}(x ; h), \Theta_{1}^{T}\left(\mathbf{I}_{K} \otimes \overline{\mathbf{Y}}\right) \mathbf{c}\right]=O\left(\frac{h}{N_{1} K}\right)
$$

for both $\hat{p}_{1}$ and $\hat{p}_{2}$. These are summarized, together with (A.11) and (A.12), to give the MSE expressions in Theorems 3.3 and 3.4 .

Lemma 1 Assume that $Y$ has a binomial distribution $B(N, p)$ for an intger $N \geq 2$ and $0<$ $p<1$. Then, we have for $k \geq 2$,

$$
E\left[\left(\frac{Y}{N}\right)^{k}\right]=p^{k}+\frac{k(k-1)}{2 N} p^{k-1}(1-p)+o\left(\frac{1}{N}\right),
$$

as $N \rightarrow \infty$.

Lemma 2 Assume that $\hat{v}_{i}=\hat{v}_{1 i}$ for $i=1,2, \ldots, K$. Then we have, as $N_{i} \rightarrow \infty$

$$
\begin{aligned}
E\left[\hat{v}_{i}\right] & =v_{i}-\frac{v_{i}}{N_{i}}+o\left(\frac{1}{N_{i}}\right), \\
E\left[\bar{Y}_{i} \hat{v}_{i}\right] & =p_{i} v_{i}+\frac{1}{N_{i}}\left(v_{i}-3 p_{i} v_{i}\right)+o\left(\frac{1}{N_{i}}\right), \\
E\left[\bar{Y}_{i}^{2} \hat{v}_{i}\right] & =p_{i}^{2} v_{i}+\frac{1}{N_{i}}\left(3 v_{i}^{2}-3 p_{i}^{2} v_{i}\right)+o\left(\frac{1}{N_{i}}\right),
\end{aligned}
$$




$$
\begin{aligned}
E\left[\hat{v}_{i}^{2}\right] & =v_{i}^{2}+\frac{1}{N_{i}}\left(v_{i}-6 v_{i}^{2}\right)+o\left(\frac{1}{N_{i}}\right), \\
E\left[\bar{Y}_{i} \hat{v}_{i}^{2}\right] & =p_{i} v_{i}^{2}+\frac{1}{N_{i}}\left(3 v_{i}^{2}-9 p_{i} v_{i}^{2}+p_{i}^{3} v_{i}\right)+o\left(\frac{1}{N_{i}}\right), \\
E\left[\bar{Y}_{i}^{2} \hat{v}_{i}^{2}\right] & =p_{i}^{2} v_{i}^{2}+\frac{1}{N_{i}}\left(6 v_{i}^{3}-8 p_{i}^{2} v_{i}^{2}+p_{i}^{4} v_{i}\right)+o\left(\frac{1}{N_{i}}\right),
\end{aligned}
$$

Lemma 3 Assume that $\hat{v}_{i}=\hat{v}_{2 i}$ for $i=1,2, \ldots, K$. Then we have, as $N_{i} \rightarrow \infty$

$$
\begin{aligned}
E\left[\hat{v}_{i}\right] & =v+\frac{1}{2 \sqrt{N_{i}}}\left(1-2 p_{i}\right)^{2}+\frac{1}{4 N_{i}}\left(8 v_{i}-3\right)+o\left(\frac{1}{N_{i}}\right), \\
E\left[\bar{Y}_{i} \hat{v}_{i}\right] & =p v+\frac{1}{2 \sqrt{N_{i}}} p\left(1-2 p_{i}\right)^{2}+\frac{1}{4 N_{i}}\left(4 v_{i}-3 p_{i}\right)+o\left(\frac{1}{N_{i}}\right), \\
E\left[\bar{Y}_{i}^{2} \hat{v}_{i}\right] & =p_{i}^{2} v_{i}+\frac{1}{2 \sqrt{N_{i}}} p_{i}^{2}\left(1-2 p_{i}\right)^{2}+\frac{3}{4 N_{i}}\left(4 v_{i}^{2}-p_{i}^{2}\right)+o\left(\frac{1}{N_{i}}\right), \\
E\left[\hat{v}_{i}^{2}\right] & =v_{i}^{2}+\frac{1}{\sqrt{N_{i}}} v_{i}\left(1-2 p_{i}\right)^{2}+\frac{1}{4 N_{i}}\left(1-5 v_{i}+8 v_{i}^{2}\right)+o\left(\frac{1}{N_{i}}\right), \\
E\left[\bar{Y}_{i} \hat{v}_{i}^{2}\right] & =p_{i} v_{i}^{2}+\frac{1}{\sqrt{N_{i}}} p_{i} v_{i}\left(1-2 p_{i}\right)^{2}+\frac{1}{4 N_{i}}\left(p_{i}-14 p_{i} v_{i}+4 p_{i}^{3} v_{i}+12 v_{i}^{2}+4 p_{i} v_{i}^{2}\right)+o\left(\frac{1}{N_{i}}\right), \\
E\left[\bar{Y}_{i}^{2} \hat{v}_{i}^{2}\right] & =p_{i}^{2} v_{i}^{2}+\frac{1}{\sqrt{N_{i}}} p_{i}^{2} v_{i}\left(1-2 p_{i}\right)^{2}+\frac{1}{4 N_{i}}\left(p_{i}^{2}-14 p_{i}^{2} v_{i}+4 p_{i}^{4} v_{i}+8 p_{i}^{2} v_{i}^{2}+24 v_{i}^{3}\right)+o\left(\frac{1}{N_{i}}\right) .
\end{aligned}
$$

Lemma 4 Assume that $f$ has continuous second order derivatives on $[0,1]$. Let the kernel $\phi$ be as in Assumption 3. Then we have

$$
\frac{1}{K h} \sum_{i=1}^{K} f\left(x_{i}\right) \phi\left(\frac{x_{i}-x}{h}\right)=\frac{1}{h} \int_{0}^{1} f(u) \phi\left(\frac{u-x}{h}\right) d u+O\left(\frac{1}{K h}\right) .
$$

Proof of Lemma 4 Since $\phi$ has support on $[-1,1]$ and $\phi \in C^{2}(-1,1)$,

$$
\begin{aligned}
\phi\left(\frac{u-x}{h}\right) & >0, u \in[x-h, x+h] \\
& =0, u \notin[x-h, x+h],
\end{aligned}
$$

and $\phi((u-x) / h) \in C^{2}(x-h, x+h)$. It follows

$$
\frac{1}{h} \int_{0}^{1} f(u) \phi\left(\frac{u-x}{h}\right) d u=\frac{1}{h} \int_{x-h}^{x+h} f(u) \phi\left(\frac{u-x}{h}\right) d u .
$$

Note that $\phi\left(\left(x_{i}-x\right) / h\right)=0$ for $x_{i} \notin[x-h, x+h]$. Let $k=k(h, K)$ be the number of $x_{i}$ 's contained in $[x-h, x+h]$. Since $x_{i}=(i-1) /(K-1)$,

$$
k=\left[\frac{2 h}{1 /(K-1)}\right]+1=[2(K-1) h+1]
$$


where $[t]$ is the largest integer less than $t$. Set

$$
y_{j}=(x-h)+j g, j=1,2, \ldots, k,
$$

where $g=2 h / k$. Let $I=\max \left\{i ; x_{i}<x-h\right\}$. From $(I-1) /(K-1) \leq x-h$, we have $I=[(x-h)(K-1)+1]$. Since $x_{I+1}, \ldots, x_{I+k} \in[x-h, x+h]$,

$$
\frac{1}{(K-1) h} \sum_{i=1}^{K} f\left(x_{i}\right) \phi\left(\frac{x_{i}-x}{h}\right)=\frac{1}{(K-1) h} \sum_{j=1}^{k} f\left(x_{I+j}\right) \phi\left(\frac{x_{I+j}-x}{h}\right) .
$$

By using the Euler-Maclaurin's formula [see, Yamamoto (1976)] , the equation

$$
\frac{g}{h} \sum_{j=1}^{k} f\left(y_{j}\right) \phi\left(\frac{y_{j}-x}{h}\right)=\frac{1}{h} \int_{x-h}^{x+h} f(u) \phi\left(\frac{u-x}{h}\right) d u+O\left(\frac{g}{h}\right)
$$

holds. From (A.13),(A.14),(A.15), It is enough if the following formula is shown in order to prove the lemma.

$$
\frac{1}{(K-1) h} \sum_{j=1}^{k} f\left(x_{I+j}\right) \phi\left(\frac{x_{I+j}-x}{h}\right)-\frac{g}{h} \sum_{j=1}^{k} f\left(y_{j}\right) \phi\left(\frac{y_{j}-x}{h}\right)=O\left(\frac{1}{K h}\right) .
$$

Put

$$
\begin{aligned}
\tau_{1} & =\left|\frac{1}{(K-1) h} \sum_{j=1}^{k} f\left(x_{I+j}\right) \phi\left(\frac{x_{I+j-x}-x}{h}\right)-\frac{1}{(K-1) h} \sum_{j=1}^{k} f\left(y_{j}\right) \phi\left(\frac{y_{j}-x}{h}\right)\right|, \\
\tau_{2} & =\left|\left\{\frac{1}{(K-1) h}-\frac{g}{h}\right\} \sum_{j=1}^{k} f\left(y_{j}\right) \phi\left(\frac{y_{j}-x}{h}\right)\right| .
\end{aligned}
$$

Then we obtain

$$
\left|\frac{1}{(K-1) h} \sum_{j=1}^{k} f\left(x_{I+j}\right) \phi\left(\frac{x_{I+j-x}-x}{h}\right)-\frac{g}{h} \sum_{j=1}^{k} f\left(y_{j}\right) \phi\left(\frac{y_{j}-x}{h}\right)\right| \leq \tau_{1}+\tau_{2} .
$$

Noting $\left|x_{I+j}-y_{j}\right| \leq g$, it follows that

$$
\begin{aligned}
\tau_{1} & \leq \frac{1}{(K-1) h}\left|\sum_{j=1}^{k}\left(x_{I+j}-y_{j}\right) \frac{d}{d u} f(u) \phi\left(\frac{u-x}{h}\right)\right|_{u=\xi_{j}} \mid \\
& \leq \frac{1}{(K-1) h} \sum_{j=1}^{k}\left|x_{I+j}-y_{j}\right| \frac{M_{1}}{h} \\
& \leq \frac{k g}{(K-1) h} \frac{M_{1}}{h} \\
& =\frac{2}{(K-1) h} M_{1}
\end{aligned}
$$


where we used the evaluation that

$$
\sup _{u \in[0,1]}\left|\frac{d}{d u} f(u) \phi\left(\frac{u-x}{h}\right)\right| \leq \frac{M_{1}}{h},
$$

for some positive constant $M_{1}$ whose existence is implied by boundedness of $f, f^{\prime}, \phi$ and $\phi^{\prime}$. Therefore

$$
\tau_{1}=O\left(\frac{1}{K h}\right)
$$

On the other hand,

$$
\begin{aligned}
\tau_{2} & =\left|\frac{1}{(K-1) g}-1\right|\left|\frac{g}{h} \sum_{j=1}^{k} f\left(y_{j}\right) \phi\left(\frac{y_{j}-x}{h}\right)\right| \\
& =\left|\frac{k}{2(K-1) h}-1\right|\left|\frac{1}{h} \int_{x-h}^{x+h} f(u) \phi\left(\frac{u-x}{h}\right) d u+O\left(\frac{g}{h}\right)\right| \\
& =\left|\frac{[2(k-1) h]+1}{2(K-1) h}-1\right|\left|\int_{-1}^{1} f(x+h z) \phi(z) d z+O\left(\frac{1}{k}\right)\right| \\
& \leq \frac{1}{2(K-1) h}\left|\int_{-1}^{1} f(x+h z) \phi(z) d z+O\left(\frac{1}{K h}\right)\right|
\end{aligned}
$$

Hence

$$
\tau_{2}=O\left(\frac{1}{K h}\right)
$$

It follows (A.16) from (A.17) and (A.18). 


\section{ACKNOWLEDGEMENT}

Research of K. Naito was partially supported by Japan Society for the Promotion of Science 13780174 .

\section{References}

[1] Berkson, J. (1944). Application of the logistic function to bioassay. J. Amer. Statist. Assoc., 39, 357-365.

[2] Berkson, J. (1955). Maximum likelihood and minimum $\chi^{2}$ estimates of the logistic function. J. Amer. Statist. Assoc., 50, 130-162.

[3] Bliss, C. I. (1944). The method of probits. Science, 79, 38-39.

[4] Copas, J. B. (1983). Plotting $p$ against $x$. Applied Statistics 32, 25-31.

[5] Fan, J. Q., Heckman, N. E. and Wand, M. P. (1995). Local polynomial kernel regression for generalized linear models and quasi-likelihood functions. J. Amer. Statist. Assoc., 90, 141-150.

[6] Green, P. J. and Silverman, B. W. (1994). Nonparametric Regression and Generalized Linear Models. Chapman and Hall.

[7] Härdle, W. (1990). Applied Nonparametric Regression. Cambrige University Press.

[8] Lloyd, C. J. (1999). Statistical Analysis of Categorical Data. Wiley, New York.

[9] Müller, H.-G. (1985). Kernel estimators of zeros and of location and size of extrema of regression functions. Scand. J. Statist., 12, 221-232.

[10] Müller, H.-G. and Schmitt, T. (1988). Kernel and probit estimates in Quantal Bioassay. J. Amer. Statist. Assoc., 83, 750-759.

[11] Nadaraya, E. A. (1964). On estimating regression. Theory Probab. Appl., 9 141-142.

[12] Ruppert, D. and Wand, M. P. (1994). Multivariate locally weighted least squares regression. Ann. Statist., 22, 1346-1370.

[13] Silvapulle, M. J. (1981). On the existence of maximum likelihood estimators for the binomial response model. J. Roy. Statist. Soc. B,43, 310-313.

[14] Simonoff, J. S. (1996). Smoothing methods in statistics. Springer.

[15] Staniswalis, J. G. and Cooper, V. (1988). Kernel estimates of dose response. Biometrics, 44, 1103-1119.

[16] Wand, M. P. and Jones, M. C. (1995). Kernel Smoothing. Chapman and Hall.

[17] Watson, G. S. (1964). Smooth regression analysis. Sankhyā Ser.A, 26 101-116. 
[18] Yamamoto, T. (1976). Suuchi kaiseki nyuumon(Introduction to Numerical Analysis). Saiensusha(in Japanese). 


\section{Table Captions}

Caption for Table 1: The optimal MPEs of $\hat{p}_{1}, \hat{p}_{2}, \hat{p}_{N W}$ and $\hat{p}_{L}$ for $p(x)=p_{\text {logit }}(x ; 4,8), K=5$, and $N_{i}=150,200$, and 250. The value in the parenthesis is the bandwidth that gives the optimal MPE.

Caption for Table 2: The optimal MPEs of $\hat{p}_{1}, \hat{p}_{2}, \hat{p}_{N W}$ and $\hat{p}_{L}$ for $p(x)=p_{\text {logit }}(x ; 4,2), K=5$, and $N_{i}=150,200$, and 250. The value in the parenthesis is the bandwidth that gives the optimal MPE.

Caption for Table 3: The optimal MPEs of $\hat{p}_{1}, \hat{p}_{2}, \hat{p}_{3}, \hat{p}_{4}, \hat{p}_{N W}$, and $\hat{p}_{\mathrm{MLE}}$ for $p(x)=$ $p_{\text {logit }}(x ; 4,8), K=5$, and $N_{i}=5,10$, and 15 , where $\hat{p}_{\text {MLE }}$ is the MLE based on the logistic model. The value in the parenthesis is the bandwidth that gives the optimal MPE.

Caption for Table 4: The optimal MPEs of $\hat{p}_{1}, \hat{p}_{2}, \hat{p}_{3}, \hat{p}_{4}, \hat{p}_{N W}$, and $\hat{p}_{\mathrm{MLE}}$ for $p(x)=$ $p_{\text {logit }}(x ; 4,2), K=5$, and $N_{i}=5,10$, and 15 , where $\hat{p}_{\text {MLE }}$ is the MLE based on the logistic model. The value in the parenthesis is the bandwidth that gives the optimal MPE. 
Table 1

\begin{tabular}{clll}
\hline$N_{i}$ & 150 & 200 & 250 \\
\hline $\operatorname{MPE}\left[\hat{p}_{1}\right]$ & $299(.088)$ & $227(.086)$ & $183(.085)$ \\
$\operatorname{MPE}\left[\hat{p}_{2}\right]$ & $325(.070)$ & $244(.070)$ & $195(.070)$ \\
$\operatorname{MPE}\left[\hat{p}_{N W}\right]$ & $310(.096)$ & $236(.092)$ & $190(.009)$ \\
$\operatorname{MPE}\left[\hat{p}_{L}\right]$ & 330 & 248 & 198 \\
\hline
\end{tabular}


Table 2

\begin{tabular}{clll}
\hline$N_{i}$ & 150 & 200 & 250 \\
\hline $\operatorname{MPE}\left[\hat{p}_{1}\right]$ & $119(.146)$ & $93(.140)$ & $76(.136)$ \\
$\operatorname{MPE}\left[\hat{p}_{2}\right]$ & $121(.145)$ & $93(.140)$ & $76(.137)$ \\
$\operatorname{MPE}\left[\hat{p}_{N W}\right]$ & $105(.187)$ & $84(.173)$ & $70(.164)$ \\
$\operatorname{MPE}\left[\hat{p}_{L}\right]$ & 178 & 133 & 107 \\
\hline
\end{tabular}




\section{Table 3}

\begin{tabular}{clll}
\hline$N_{i}$ & 5 & 10 & 15 \\
\hline $\operatorname{MPE}\left[\hat{p}_{1}\right]$ & $3894(.185)$ & $2928(.197)$ & $2399(.114)$ \\
$\operatorname{MPE}\left[\hat{p}_{2}\right]$ & $3968(.181)$ & $2464(.174)$ & $1766(.169)$ \\
$\operatorname{MPE}\left[\hat{p}_{3}\right]$ & $12098(.139)$ & $4737(.137)$ & $2908(.121)$ \\
$\operatorname{MPE}\left[\hat{p}_{4}\right]$ & $12527(.130)$ & $5290(.135)$ & $3133(.136)$ \\
$\operatorname{MPE}\left[\hat{p}_{N W}\right]$ & $4619(.167)$ & $3015(.153)$ & $2232(.144)$ \\
$\operatorname{MPE}\left[\hat{p}_{\text {MLE }}\right]$ & 4638 & 2489 & 1679 \\
\hline MLE's existence probability & .75263 & .94479 & .98712 \\
\hline
\end{tabular}


Table 4

\begin{tabular}{rlll}
\hline$N_{i}$ & 5 & 10 & 15 \\
\hline $\operatorname{MPE}\left[\hat{p}_{1}\right]$ & $266(.544)$ & $423(.344)$ & $552(.266)$ \\
$\operatorname{MPE}\left[\hat{p}_{2}\right]$ & $341(.569)$ & $401(.405)$ & $457(.334)$ \\
$\operatorname{MPE}\left[\hat{p}_{3}\right]$ & $6326(.648)$ & $2459(.518)$ & $1159(.424)$ \\
$\operatorname{MPE}\left[\hat{p}_{4}\right]$ & $6660(.605)$ & $2979(.479)$ & $1567(.409)$ \\
$\operatorname{MPE}\left[\hat{p}_{N W}\right]$ & $718(.569)$ & $534(.433)$ & $499(.377)$ \\
$\operatorname{MPE}\left[\hat{p}_{\text {MLE }}\right]$ & 3837 & 1277 & 821 \\
\hline MLE's existence probability & .36027 & .71147 & .88276 \\
\hline
\end{tabular}




\section{Figure Captions}

Caption for Figure 1: True curves $p(x)=p_{\text {logit }}(x ; 4,8)$; solid line, and $p_{\text {logit }}(x ; 4,2)$; dashed line.

Caption for Figure 2: MSEs of estimators against $x$ for the curve $p(x)=p_{\text {logit }}(x ; 4,8), K=5$ and $N_{i}=200$.

Caption for Figure 3: MSEs of estimators against $x$ for the curve $p(x)=p_{\text {logit }}(x ; 4,2), K=5$ and $N_{i}=200$.

Caption for Figure 4: Curves $p(x)=0.2 \sin (2 \pi x)+0.5$; solid line, and $0.4 \sin (2 \pi x)+0.5$; dashed line.

Caption for Figure 5: The function $g(x)$ for $p(x)=0.2 \sin (2 \pi x)+0.5$; solid line, and for $p(x)=0.4 \sin (2 \pi x)+0.5 ;$ dashed line.

Caption for Figure 6: MSEs of estimators for the curve $p(x)=p_{\text {logit }}(x ; 4,8), K=5$ and $N_{i}=10$.

Caption for Figure 7: MSEs of estimators for the curve $p(x)=p_{\text {logit }}(x ; 4,2), K=5$ and $N_{i}=10$. 
Figure 1

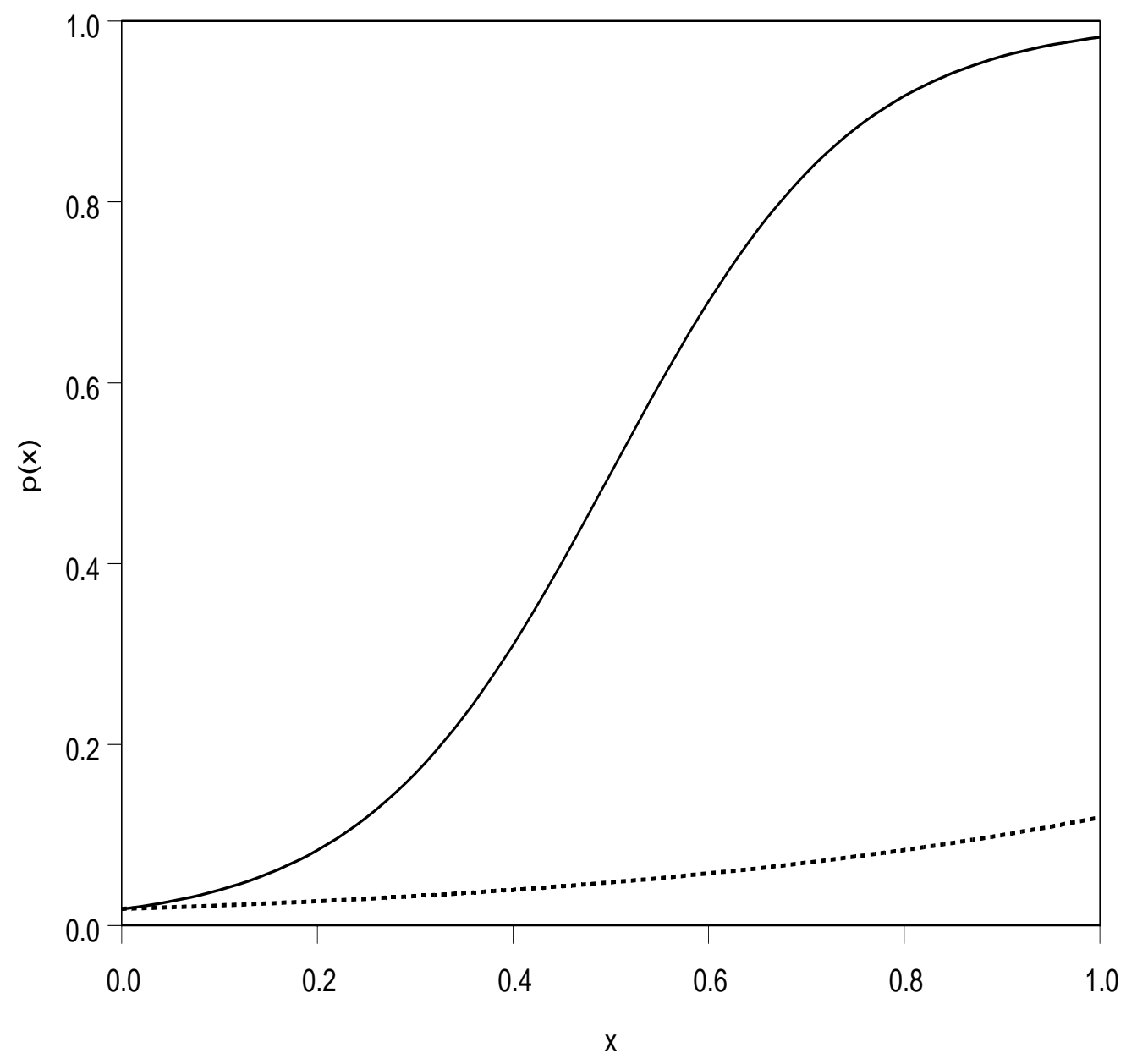


Figure 2

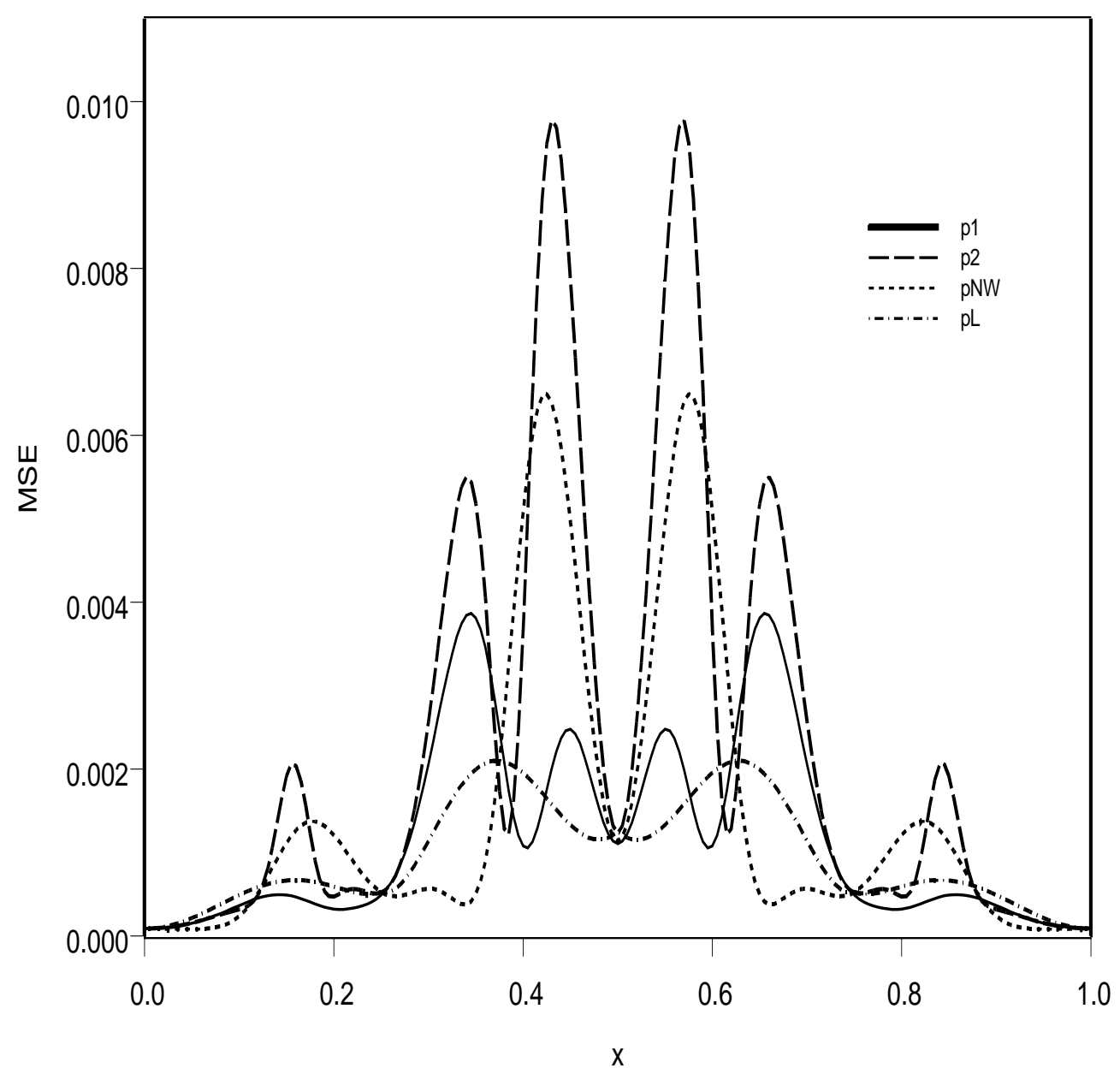


Figure 3

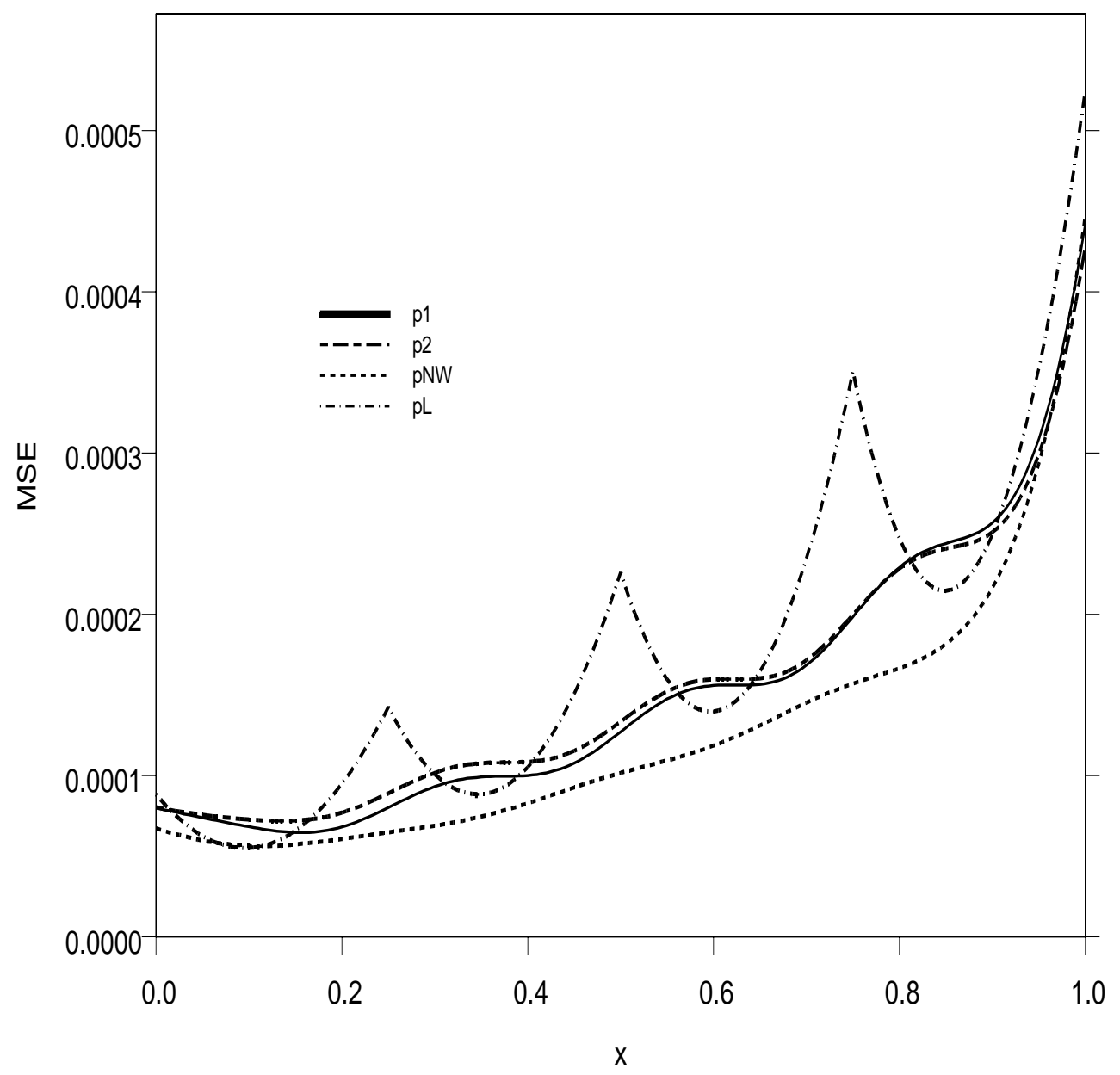


Figure 4

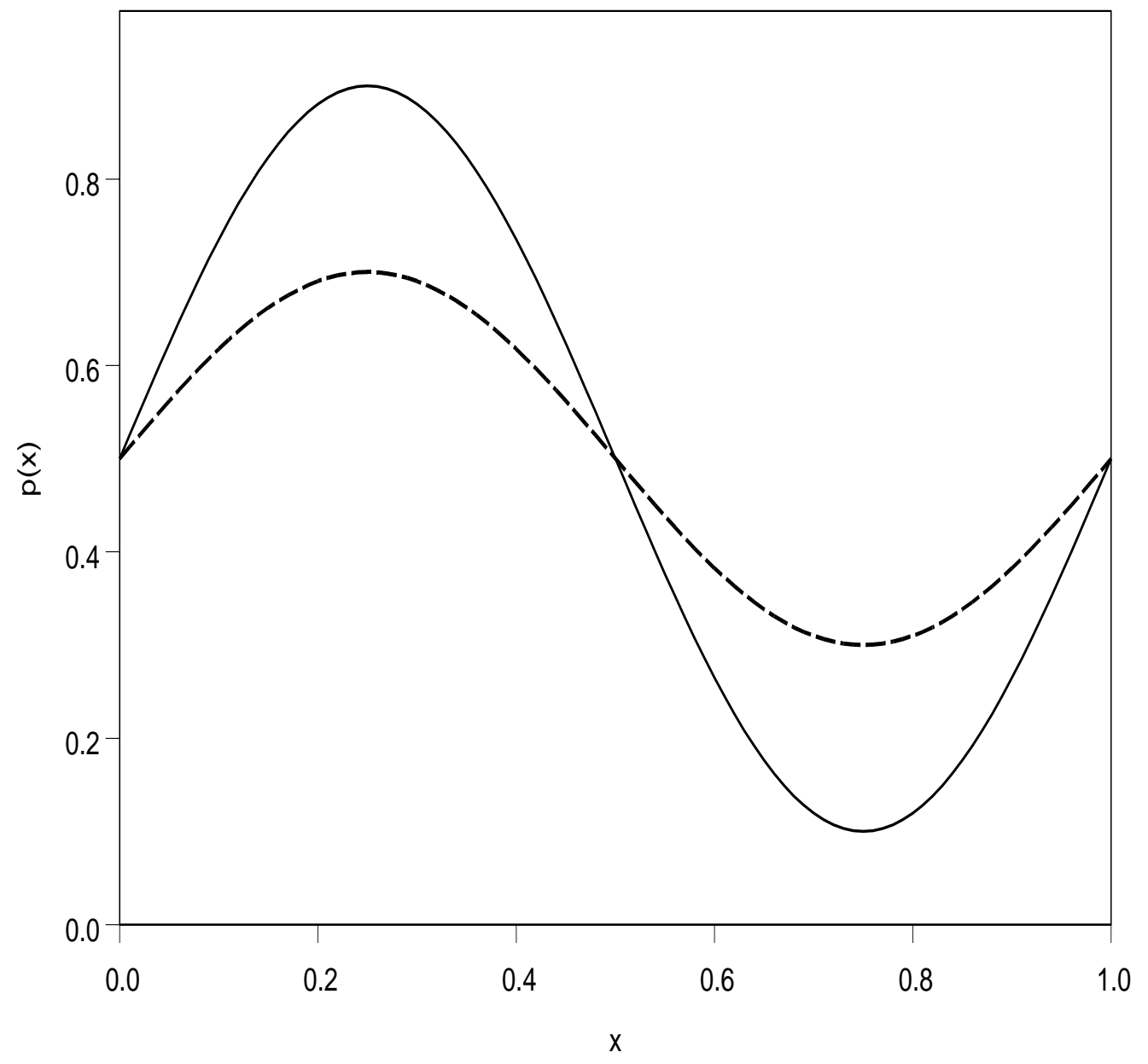


Figure 5

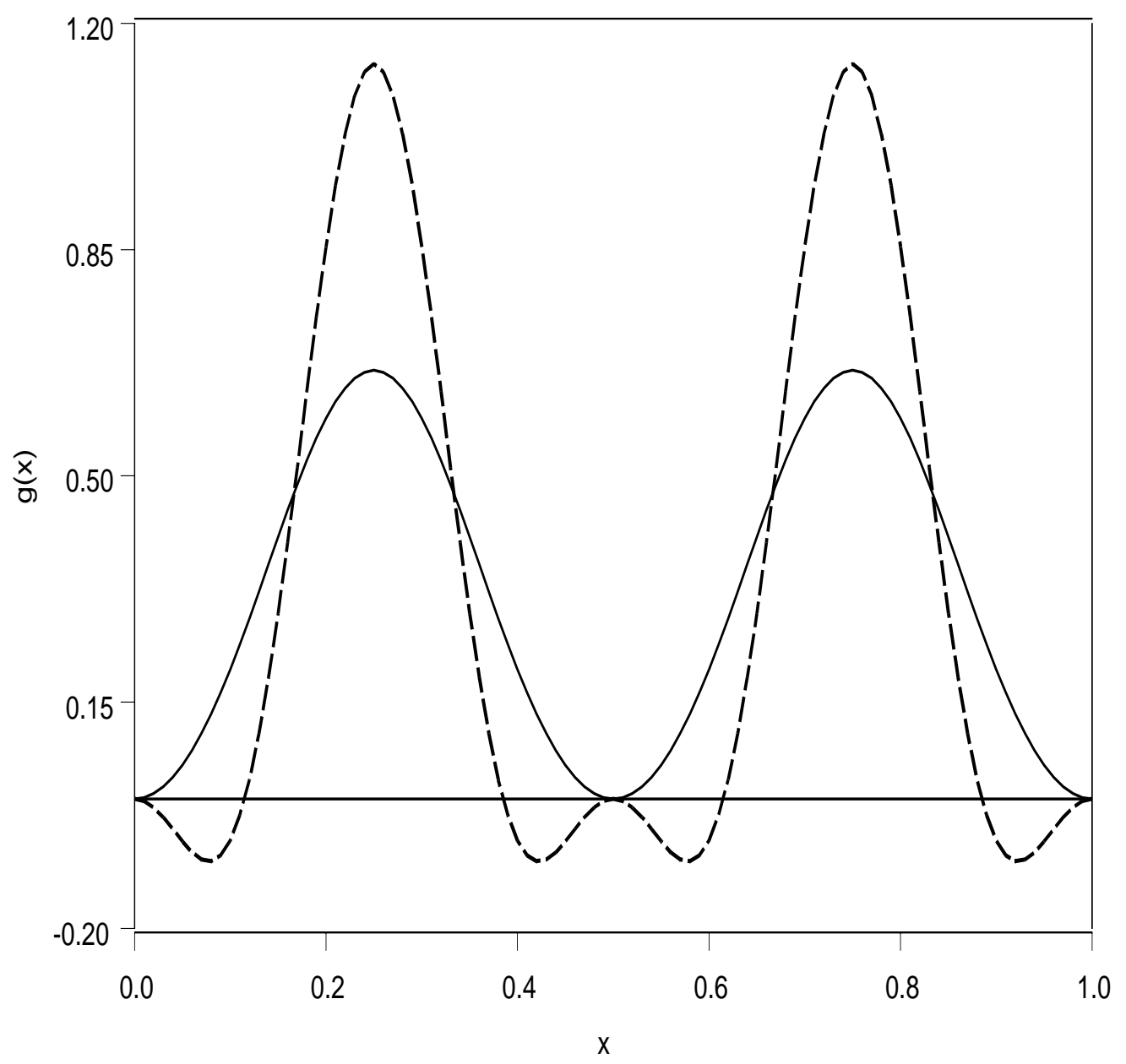


Figure 6

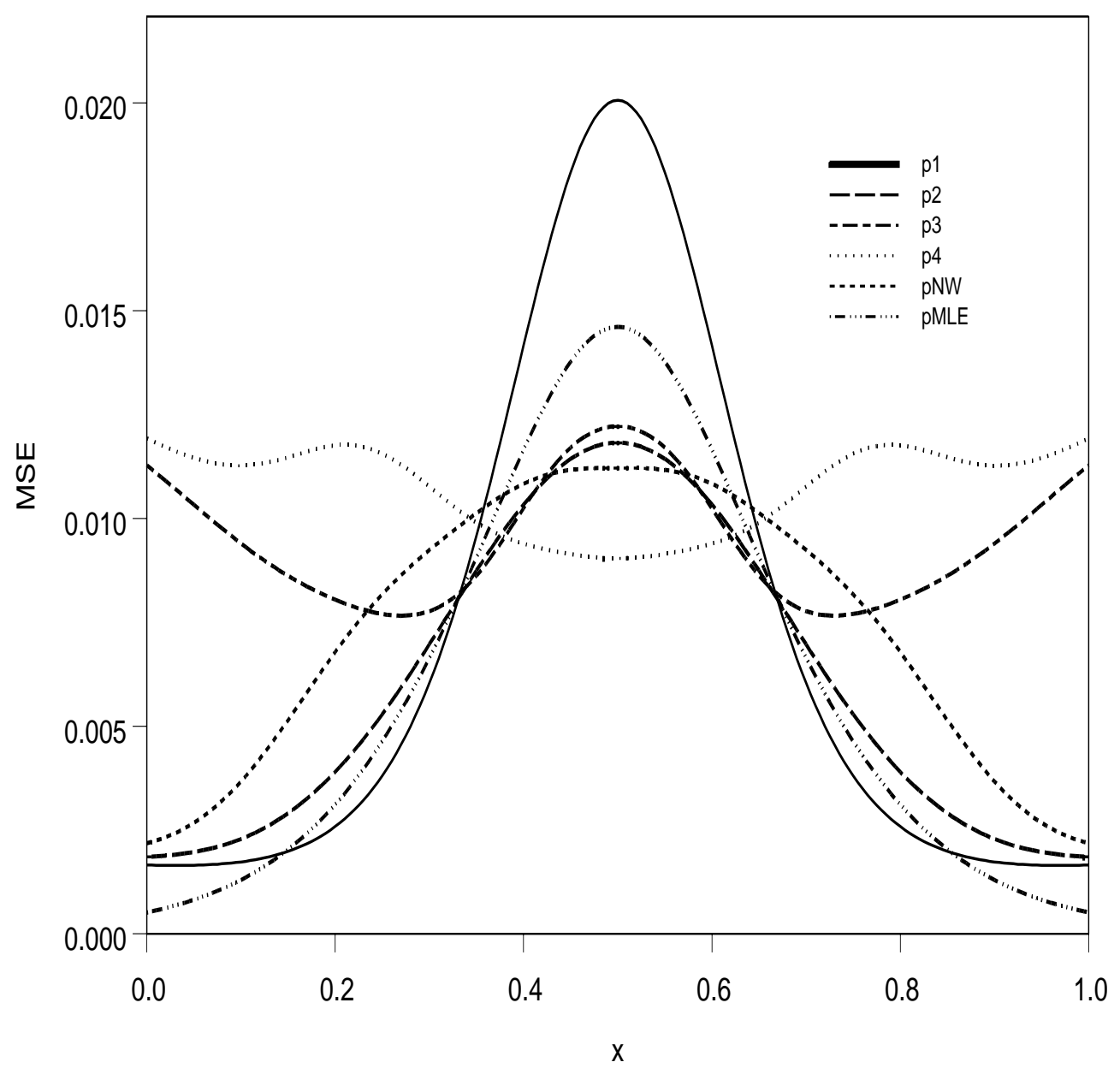


Figure 7

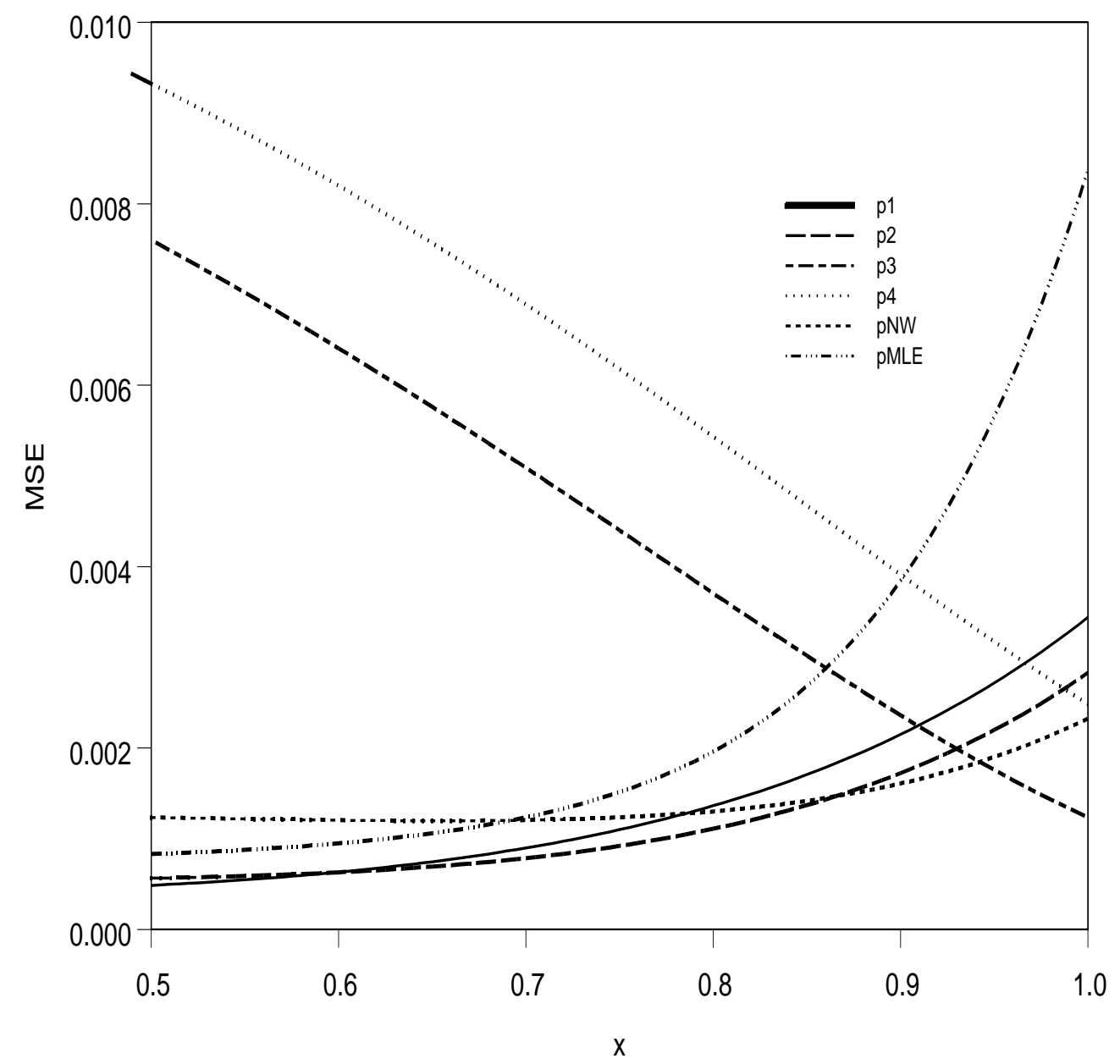

